\title{
The Operator Algebra of Orbifold Models
}

\author{
Robbert Dijkgraaf ${ }^{1}$, Cumrun Vafa ${ }^{2}$, Erik Verlinde ${ }^{3}$, and Herman Verlinde ${ }^{4}$ \\ 1 Institute for Theoretical Physics, University of Utrecht, P. O. Box 80.006, \\ NL-3508 TA Utrecht, The Netherlands \\ 2 Lyman Laboratory of Physics, Harvard University, Cambridge, MA 02138, USA \\ 3 Institute for Advanced Study, Princeton, NJ 08540, USA \\ 4 Joseph Henry Laboratories, Princeton University, Princeton, NJ 08544, USA
}

\begin{abstract}
We analyze the chiral properties of (orbifold) conformal field theories which are obtained from a given conformal field theory by modding out by a finite symmetry group. For a class of orbifolds, we derive the fusion rules by studying the modular transformation properties of the one-loop characters. The results are illustrated with explicit calculations of toroidal and $c=1$ models.
\end{abstract}

\section{Introduction}

The classification of conformal invariant field theories has up to now only been exhaustive for $c<1$ [1-4]. This is due to the particular properties of the Virasoro algebra for these values of the central charge. It only has a finite set of unitary representations and only for these minimal models the Virasoro algebra gives enough information - in the form of null states - to determine the possible interactions. It has long been evident that extensions of the conformal symmetry are needed in order to give equally exhaustive results in the classification for $c \geqq 1$. Partial results in this direction have been obtained, mainly in the context of superconformal and affine Lie algebras.

There has recently been much interest in rational conformal field theories (RCFT's) [5-13]. These theories are characterized by the fact that their correlations are given by a finite sum of holomorphic times anti-holomorphic functions of the moduli of punctured surfaces. This property of the RCFT's can be used to derive powerful constraints on the operator content and operator product relations in these models. In this respect they form a natural generalization of the minimal theories and it is clearly an important problem to try and find a complete description of all possible RCFT's. From this viewpoint, it would be very interesting to devise operations that act on the space of RCFT's and generate new theories from a given one. As an example one can think of the construction of nondiagonal modular invariant combinations of characters, coset models [14], etc. Another operation of that kind is the concept of an orbifold. 
The original motivation of studying orbifolds [15] was to obtain simple models of string compactification, which are more or less realistic. The simplext orbifold models are constructed out of tori with certain isometries. One considers the string propagation on the quotient of the torus by some subgroup of its symmetry group to obtain a new theory, which is only slightly more complicated to analyze than the toroidal model we started with. In general, the operation of taking the quotient of a space by its symmetry leads to singular points if the symmetry operation has fixed points. The singularity of the space would render point particle theories on such spaces inconsistent. However string theory manages to avoid this problem by the introduction of string states which are closed only up to the action of the group element, i.e. by enlarging the Hilbert space to include twisted string states. These extensions are known as the twisted sectors.

The whole idea of orbifolds can be applied to general conformal theories with discrete symmetries. Given any CFT $\mathscr{C}$ with a particular symmetry group $G$, one could in principle try to construct a CFT $\mathscr{C} / G$ where one imposes an equivalence relation $\bmod G$ on the full theory. Again the main new feature of the theory $\mathscr{C} / G$ is the introduction of twisted Hilbert space sectors. We shall continue to call such conformal theories orbifold models.

Due to a lack of a geometrical understanding of the twisted states for a general conformal theory, the investigation of abstract orbifold theories has had only a limited amount of progress. Some of the properties of general orbifolds, such as the partition functions for twisted sectors can only be deduced by the requirement of modular invariance. This is in sharp contrast to toroidal orbifolds where we can a priori determine the partition function of the twisted sectors by geometric arguments and that always turns out to be consistent with modular invariance.

In this paper we will investigate some general properties of orbifold theories. In particular we will study the operator algebra and the role of the modular group. The abstract setting that we shall follow permits for quite general orbifolds, and not just the toroidal ones. It is pleasant to find that the basic structures of orbifolds are for a large part dictated by the group structure and depend relatively little on what the underlying conformal field theory is. This is particularly true for the case of orbifolds constructed from so-called holomorphic conformal theories, i.e. theories for which all operators are contained in the chiral algebra. We will show that for this class of orbifolds the modular geometry can be described entirely in terms of the finite group, up to certain phases. The reason that even in these holomorphic theories more structure appears than can be obtained from the finite group lies in the fact that we are interested in the chiral group action. So one has to split the Hilbert space into chiral blocks, and there is some additional information about the underlying CFT in the form of how this left-right splitting is accomplished.

The organization of this paper is as follows. We start in Sect. 2 with a brief review of rational conformal field theory. In Sect. 3 we derive, using general arguments, the operator content and the possible form of interactions in orbifold theories. Here we will mainly discuss orbifolds of holomorphic conformal field theories. These arguments will be substantiated in Sect. 4 where the formulation of orbifolds on the torus is discussed. Using the modular properties of these theories we will derive the fusion rules and conformal dimensions of the operators. We also 
discuss briefly higher genus surfaces and discrete torsion. The results are applied and illustrated in some examples in Sect. 5. In Sect. 6 we consider the much more difficult case of non-holomorphic models. We will make a start in characterizing this general class of orbifolds, but our results are not yet complete. As an illustration we will discuss in Sect. 7 in detail the interactions in the various $c=1$ orbifold models. Finally, in the appendix we have collected some useful identities obtained in the theory of finite groups.

\section{Rational Conformal Field Theories and Their Fusion Algebras}

For any conformal theory one can decompose the Hilbert space into the representations of left and right Virasoro algebras Vir and Vir. What is special about RCFT's is that their Hilbert space decomposes to a finite sum of representations (not necessarily irreducible)

$$
\mathscr{H}=\sum_{(i, i)}\left[\phi_{i}\right] \otimes\left[\bar{\phi}_{i}\right]
$$

where $\left[\phi_{i}\right]$ denotes a representation of Vir and $\left[\bar{\phi}_{\bar{i}}\right]$ denotes a representation of $\overline{\mathrm{Vir}}$. From the fact that $L_{0}-\bar{L}_{0}$ has integer spectrum, we easily deduce that all the states in a given subsector $\left[\phi_{i}\right]$ have the same eigenvalue $h_{i}$ of $L_{0}$ modulo addition of an integer.

The fact that the subsectors are not necessarily irreducible representations of the Virasoro algebra (and for $c \geqq 1$ they are necessarily not so [3]) motivates us to look for some kind of an extension of the Virasoro algebra for which the subsectors $\left[\phi_{i}\right]$ (and similarly for $\left[\bar{\phi}_{i}\right]$ ) do form irreducible representations. The investigation of such extended algebras has only started recently, and so far complete results have been established only for certain special cases such as the minimal models and the Kac-Moody algebras. In these cases there is a finite number of operators which generate the rest of the algebra by taking various combinations. In the case of Kac-Moody the generators are the vertex operators of the spin one currents $J^{a}(z)$, and in the Virasoro case it is the vertex operator for the spin two state corresponding to $T(z)$, i.e. the energy momentum tensor. Due to the fact that in these two cases we actually obtain (affine) Lie algebras for the algebra of Fourier components of these vertex operators, it is possible to develop a representation theory of such algebras and obtain a complete classification of the irreducible representations of the algebra. For general chiral algebras, however, much less is known. In particular, it is not known whether there will always exist a finite set of generators, and if so whether it leads to a simple enough algebra to yield a classification of its irreducible representations. Even so, it turns out to be very convenient to introduce the notion of such a chiral algebra, and we will proceed to do so, without ever making use of its explicit form.

Our definition of the chiral algebra of a general RCFT is based on the decomposition (2.1) of the Hilbert space. Among the chiral sectors $\left[\phi_{i}\right]$ there is precisely one sector which includes the vacuum state. We will denote this sector by $\left[\phi_{0}\right]$. We can now consider all the vertex operators corresponding to states in $\left[\phi_{0}\right]$, which we shall denote by $\mathscr{A}$ and call the chiral algebra of the RCFT. All the 
operators in $\mathscr{A}$ have integral conformal dimensions, similar to the Kac-Moody and the Virasoro case. It is useful to introduce this chiral algebra $\mathscr{A}$ because there is an intimate relation between the interactions in a conformal field theory and the representation theory of $\mathscr{A}$. To explain this relation, let us first recall that all information about the interactions in a CFT is contained in the three point functions. So the central object in a CFT is the state it assigns to a sphere with three punctures (together with coordinates at the punctures), or equivalently to a pant or trinion. This state will be denoted by $|V\rangle$ and is called the three point vertex (which we take to correspond to the sphere $\mathbf{P}^{1}$ with three punctures at $0,1, \infty$ and coordinates $z, z-1,1 / z$ at the punctures)

$$
|V\rangle \in \mathscr{H} \otimes \mathscr{H} \otimes \mathscr{H}
$$

where $\mathscr{H}$ is the Hilbert space of all operators in the theory. The vertex operators corresponding to states are defined by

$$
\left\langle\phi_{1}, \phi_{2}, \phi_{3} \mid V\right\rangle=\left\langle\phi_{1}\left|\phi_{2}(1)\right| \phi_{3}\right\rangle \text {. }
$$

The conformal Ward identities determine the position dependence of $\phi_{2}$ and further imply that the vertex $|V\rangle$ is fully specified by its matrix elements with Virasoro primary states.

Using the form (2.1) of the Hilbert space $\mathscr{H}$ we can decompose $|V\rangle$ as follows:

$$
|V\rangle=\sum_{i, j, k} \sum_{a}\left|V_{i j k}^{(a)}\right\rangle \otimes\left|\bar{V}_{i j k}^{(a)}\right\rangle
$$

where the chiral three point vertices $V_{i j k}^{(a)}$ satisfy

$$
\left|V_{i j k}^{(a)}\right\rangle \in\left[\phi_{i}\right] \otimes\left[\phi_{j}\right] \otimes\left[\phi_{k}\right]
$$

where $a$ runs from $1, \ldots, N_{i j k}$. The integer $N_{i j k}$ therefore denotes the different ways the chiral sectors $i, j, k$ could be coupled to their anti-holomorphic partners. Note that by permutation properties of the vertex $N_{i j k}$ is totally symmetric.

One of the basic properties of $N_{i j k}$ is that

$$
N_{i j 0}=\mathscr{C}_{i j}
$$

with $\mathscr{C}$ the charge conjugation operator. This property follows from the fact that the vacuum state is in the $\left[\phi_{0}\right]$ Hilbert space, and that the vacuum state is represented by the identity operator. In this way we discover that since all the operators in $\mathscr{A}$ happen to be in the same chiral block as the vacuum, they must also possess this symmetry. Namely, if $A(z)$ denotes an operator in $\mathscr{A}$, we must have

$$
A(z)\left[\phi_{i}\right] \subset\left[\phi_{i}\right]
$$

in other words the Hilbert subsectors $\left[\phi_{i}\right]$ form representations of the $\mathscr{A}$ algebra. Note that the chiral operators in $\left[\phi_{i}\right]$ are all local with respect to $\mathscr{A}$ but not necessarily to the operators in another representation $\left[\phi_{j}\right]$. Of course, the operators occurring in the complete non-chiral model are all mutually local.

It is a non-trivial result, proved in $[10,11]$, that the chiral sectors $\left[\phi_{i}\right]$ are actually the complete set of irreducible representations of the $\mathscr{A}$ algebra. This 
result allows us to characterize the chiral couplings $V_{i j k}^{(a)}$ in terms of the representation theory of $\mathscr{A}$ as the set of $\mathscr{A}$-invariant elements of $\left[\phi_{i}\right] \otimes\left[\phi_{j}\right] \otimes\left[\phi_{k}\right]$. Here, by $\mathscr{A}$-invariant we mean that the three point functions constructed out of $V_{i j k}^{(a)}$ satisfy the Ward identities associated with the chiral algebra $\mathscr{A}$. The integers $N_{i j k}$ give the number of independent chiral couplings, which can be interpreted as the Clebsch-Gordan coefficients of $\mathscr{A}$. Given these integers $N_{i j k}$ we can define the fusion algebra as the abstract commutative, associative algebra with unity, whose elements $\phi_{i}$ satisfy

$$
\phi_{i} \times \phi_{j}=\sum_{k} N_{i j}{ }^{k} \phi_{k}
$$

Here and in the subsequent, indices are raised with the charge conjugation matrix $\mathscr{C}$. Associativity is a straightforward consequence of duality. This algebra can be viewed as the representation algebra of the chiral algebra $\mathscr{A}$.

\section{Rational Orbifold Models}

We will now consider RCFT's that allow for the action of a finite group $G$ and investigate the operator algebra of the new orbifold CFT obtained by dividing out the symmetry $G$. In order to get a consistent model, $G$ has to be a subgroup of the group of endomorphisms of the operator algebra, and respect the left-right decomposition (2.1) of the Hilbert space. Furthermore $G$ should commute with the Virasoro algebra. We further make the restriction to a left-right symmetric action of $G$. It is clear that the structure of the resulting orbifold theory strongly depends on the way the group of $G$ intertwines with the fusion algebra of the original model. In general the group $G$ will act on the different representations $\left[\phi_{i}\right]$ of $\mathscr{A}$ by some permutation group, which commutes with the fusion algebra coefficient $N_{i j k}$. In other words, if $g \in G$ acts on the set of indices labelling the Hilbert subsectors by $i \rightarrow g(i)$ then $N_{i j k}=N_{g(i) g(j) g(k)}$. The cases in which the group $G$ acts nontrivially on the operator algebra will be referred to as the "outer" case. The word outer is suitable for describing the action of $G$ on conformal theories which permutes the operators, because the chiral algebra, which is constructed out of states in the identity block, acts only within each chiral block. This means that in such cases the action of the group cannot be represented by the operators inside the chiral algebra we started with. The case when all elements of $G$ act trivially on the fusion algebra is called inner ${ }^{1}$.

The determination of the full representation content of a general orbifold theory can be quite complicated, especially because in general the group $G$ consists of both inner and outer automorphisms. Roughly speaking, one expects that the outer action of the group results in the identification of representations, while the inner action gives rise to splitting of representations and the emergence of twisted sectors. The aim of this work is to make this intuitive picture more precise. We will mainly concentrate on the effect of the inner action. To simplify the discussion we

\footnotetext{
${ }^{1}$ This does not necessarily mean that in the inner case the automorphism can always be represented by elements in the chiral algebra. An example of this kind is provided by $E_{8} \times E_{8}$ (both at level one) and the automorphism which exchanges the two $E_{8}$ 's
} 
first consider the case where the representation theory of chiral algebra $\mathscr{A}$ of the original model is trivial i.e. when no other irreducible representations occur, except the basic representation $\mathscr{A}$ itself. Such a model will be called a holomorphic $\mathrm{CFT}^{2}$.

\section{3.a. Holomorphic Theories: Untwisted Sector}

The Hilbert space of a holomorphic conformal theory is equal to the tensor product $\mathscr{A} \otimes \overline{\mathscr{A}}$ of the left- and right chiral algebra. Consequently, the partition function factorizes

$$
\mathscr{Z}(q, \bar{q})=\chi(q) \chi^{\prime}(\bar{q}) .
$$

Famous examples of holomorphic theories are the level 1 affine $E_{8}$ and Spin (32) $/ \mathbf{Z}_{2}$ WZW-models, or more generally models based on even self-dual lattices, and certain quotients of them (e.g. the Moonshine module [19, 20]).

We now consider a group $G$ whose elements $g$ act as endomorphisms on $\mathscr{A}$ and leave the Virasoro algebra invariant. Under the action of $G$ the chiral algebra $\mathscr{A}$ decomposes into subsectors $\mathscr{A}_{\alpha}$ containing the states that transform in the irreducible representations $r_{\alpha}$ of $G$ :

$$
\mathscr{A}=\bigoplus_{\alpha} \mathscr{A}_{\alpha} .
$$

Our aim is to construct RCFT's described by the subalgebra $\mathscr{A}_{0}$ of $\mathscr{A}$ which is invariant under the $G$ action. We will also write $\mathscr{A}_{0}=\mathscr{A} / G$. These models are the $G$-orbifold models associated with the original model with algebra $\mathscr{A}$. We will try to analyze these orbifold theories using the relation between $\mathscr{A}$ and $\mathscr{A}_{0}$. Indeed many of the properties of the $\mathscr{A}_{0}$ models only become clear once we realize that they can be obtained by this orbifold construction.

The $\mathscr{A}_{\alpha}$ form highest weight representations of $\mathscr{A}_{0}$. They are however in general not irreducible. This is due to the fact that $G$ acts within $\mathscr{A}_{\alpha}$ and commutes with the action of $\mathscr{A}_{0}$. Accordingly we have a decomposition

$$
\mathscr{A}_{\alpha}=\left[\phi_{\alpha}\right] \otimes r_{\alpha},
$$

where we want to identify $\left[\phi_{\alpha}\right]$ with an irreducible $\mathscr{A}_{0}$ representation. This can be considered a coset construction, since we identify orbits of states under $G$. Furthermore, we immediately see that $\left[\phi_{\alpha}\right]$ occurs with multiplicity $d_{\alpha}=\operatorname{dim} r_{\alpha}$. Let us stress here that $G$ does not act in the orbifold model although we label the operators with a representation index of $G$.

One should now ask whether the $\left[\phi_{\alpha}\right]$ are the only possible representations of $\mathscr{A}_{0}$. It is clear that in general this will not be the case, because we have reduced the algebra and as a consequence there will be extra operators that are local with respect to it. Concretely, since $\mathscr{A}_{0}$ is $G$ invariant, we also have to consider fields that are local with respect to $\mathscr{A}$ up to the action of an element $g \in G$. These extra fields are known as twist fields and can be organized in "twisted" representations of $\mathscr{A}_{0}$. Further, since we are considering the case for which in the original model

\footnotetext{
${ }^{2}$ Although usually the term holomorphic is reserved for a CFT $\mathscr{C}$ with only holomorphic operators, here we will use it for theories of the form $\mathscr{C} \times \overline{\mathscr{C}}$
} 
only the trivial representation of $\mathscr{A}$ occurs, it is natural to assume that these twisted representations are the only other representations of $\mathscr{A}_{0}$ (besides the $\left[\phi_{\alpha}\right]$ ). A more rigorous derivation of this operator content uses the invariance under modular transformations. We will return to that point of view in Sect. 4.

What can we say about the possible couplings of the orbifold algebra? Let us first restrict ourselves to the couplings between the untwisted representations $\left[\phi_{\alpha}\right]$ occurring in (3.3). As we will now show, these can be completely understood using the relation to the chiral algebra $\mathscr{A}$ of the original model. The starting point is that the couplings in $\mathscr{A}$ are uniquely fixed, and, since the elements of $G$ are endomorphisms of $\mathscr{A}$, they are $G$-invariant. These couplings are encoded in the (unique) chiral vertex $\left|V_{\mathscr{A}}\right\rangle \in \mathscr{A} \otimes \mathscr{A} \otimes \mathscr{A}$. Since we have the decomposition $\mathscr{A}=:_{\alpha}\left[\phi_{\alpha}\right] \otimes r_{\alpha}$ we can write

$$
\left|V_{\mathscr{A}}\right\rangle=\sum_{\alpha, \beta, \gamma} \sum_{\alpha} c_{\alpha \beta \gamma}^{(a)}\left|V_{\alpha \beta \gamma}^{(a)}\right\rangle
$$

This defines the chiral vertex operators of the $\mathscr{A}_{0}$ model

$$
\left|V_{\alpha \beta \gamma}^{(a)}\right\rangle \in\left[\phi_{\alpha}\right] \otimes\left[\phi_{\beta}\right] \otimes\left[\phi_{\gamma}\right]
$$

The $G$ invariance of $V_{\mathscr{A}}$ implies that the coefficients

$$
c_{\alpha \beta \gamma}^{(a)} \in r_{\alpha} \otimes r_{\beta} \otimes r_{\gamma}
$$

are invariant tensors of $G$, i.e. Clebsch-Gordan coefficients. Thus we see that for each independent $G$ invariant tensor $c_{\alpha \beta \text {, }}^{(a)}$ we have a corresponding $\mathscr{A}_{0}$ invariant chiral coupling $V_{\alpha \beta \gamma}^{(a)}$. Consequently the integers $N_{\alpha \beta \gamma}$ occurring in the fusion algebra of the representations $\left[\phi_{\alpha}\right]$ :

$$
\phi_{\alpha} \times \phi_{\beta}=\sum_{\gamma} N_{\alpha \beta \gamma} \phi_{\gamma}
$$

equal the number of independent Clebsch-Gordan coefficients of $G$. Hence we conclude that the fusion algebra (3.7) is identical to the representation algebra of discrete group $G$,

$$
r_{\alpha} \otimes r_{\beta}=\bigoplus_{\gamma} N_{\alpha \beta \gamma} r_{\gamma}
$$

For the holomorphic theories (3.7) represents the complete fusion algebra of representations in the untwisted sector. The above reasoning can also be applied to the identity sector of more general orbifolds to show that the representation algebra of the finite group is always realized as a subalgebra of the untwisted orbifold fusion algebra.

We can further extend the above arguments of the thrice-punctured sphere to any number of punctures. In this case one finds that the holomorphic blocks that appear in the decomposition of the correlator of a set fields $\phi_{\alpha_{2}}$ are in one-to-one correspondence with the invariant tensors Inv $\left(\otimes_{i} r_{\alpha_{i}}\right)$. This gives an explanation of the close analogy found in [12] between the tensor categories of rational conformal fields theory and group theory. In particular since the fields $\phi_{\alpha}$ have integer weights the relation $\Omega^{2}=1$ (see [12]) is obvious. 


\section{3.b. Inclusion of the Twisted Sectors}

We now turn to the couplings of the twisted sectors. Twist fields can be introduced for any element $g \in G$ and have the property that the operators in $\mathscr{A}$ have nontrivial monodromy around the twist fields given by the action of $g$. This implies in particular that they have local operator products with the chiral algebra $\mathscr{A}_{0}$. We denote the set of twisted operators associated with $g$ as $\mathscr{A}^{g}$. Note however that, since twisted boundary conditions for a non-abelian group are only uniquely defined up to conjugation, the spaces $\mathscr{A}^{g}$ only depend on the conjugacy class of $g$. We will therefore also use the notation $\mathscr{A}^{A}$ for the twisted sectors associated with the elements the conjugacy class $C_{A}$, i.e. $\mathscr{A}^{A} \equiv \mathscr{A}^{g}, g \in C_{A}$.

The twisted space $\mathscr{A}^{g}$ forms a representation of $\mathscr{A}_{0}$ but is in general not irreducible. To find the irreducible representations we proceed analogously as in the untwisted sector. Namely, within $\mathscr{A}^{g}$ there is still an action of a subgroup of $G$, viz.

$$
N_{g}=\left\{h \in G \mid[h, g]=g h g^{-1} h^{-1}=1\right\},
$$

the stabilizer group of $g$. For the elements of a particular conjugacy class $C_{A}$ the corresponding stabilizer groups are all isomorphic and we will denote this isomorphy class simply as $N_{\boldsymbol{A}}$. In an analogous way to (3.2), we decompose $\mathscr{A}^{g}$ into the irreducible representations of $\mathscr{A}_{0}$, using the action of $N_{g}$

$$
\mathscr{A}^{g}=\bigoplus_{\alpha} \mathscr{A}_{\alpha}^{g},
$$

where $\alpha$ labels the irreducible representations of $N_{g}$. Again the $\mathscr{A}_{\alpha}^{g}$ are representations of $\mathscr{A}_{0} \times G$ and can be written as

$$
\mathscr{A}_{\alpha}^{g}=\left[\phi_{\alpha}^{g}\right] \otimes r_{\alpha}^{g} .
$$

This defines the $\mathscr{A}_{0}$-modules $\left[\phi_{\alpha}^{g}\right]=\left[\phi_{\alpha}^{A}\right]$ labelled by the conjugacy class $C_{A}$ of $g$ and the irreducible representation $\alpha$ of $N_{g}$.

Let us however make the following comments. First of all, since we are dealing with chiral objects, it is possible that the chiral action of the stabilizer subgroups in the twisted sectors is projective. So we should in principle allow for a nontrivial $U(1)$ cocycle $c_{g}\left(h_{1}, h_{2}\right)$, which is common to all representations $r_{\alpha}^{g}$ in a given twisted sector. Although most of the following analysis will not depend on the presence of these cocycles, we will for convenience assume that the representations $r_{\alpha}^{g}$ are non-projective. The modifications which arise in case the stabilizer subgroups act projectively will be discussed in Sect. 4c.

A related point is the following. The labelling of the above operators is not unique. We first note that the set of operators are in one to one correspondence with the states in the Hilbert space. So the question of decomposing $\mathscr{A}^{g}$ into representations of $G$ is equivalent to defining the action of $N_{g}$ on the corresponding twisted Hilbert space $\mathscr{H}_{g}$. However, experience with orbifolds indicate that there are in general ambiguities in choosing phase assignments to the group operations in the twisted sectors. In particular the discrete torsion [18] is a reflection of such potential ambiguities. Of course the phases of operators cannot be changed arbitrarily, and they should be chosen so that the operators still satisfy the group law. Therefore, given the action of a group $N_{g}$ on a Hilbert space one has a 
fundamental ambiguity in identifying the irreducible representations by the action of a one-dimensional representation of $N_{g}$. In other words given an irreducible representation $r(h)$ of $N_{g}$ in the Hilbert space, we have the freedom to redefine $r(h) \rightarrow \varepsilon(h) r(h)$, where $\varepsilon(h)$ is a one-dimensional representation. Tensoring with $\varepsilon$ induces a permutation of the irreducible representations:

$$
r_{\alpha} \rightarrow r_{\alpha^{\prime}}=\varepsilon \otimes r_{\alpha} .
$$

It is easy to see that $r_{\alpha^{\prime}}$ is again irreducible, since $\varepsilon \otimes r_{\alpha}=r_{\alpha^{\prime}}+r_{\alpha^{\prime}}$ would imply $r_{\alpha}=\varepsilon^{*} \otimes r_{\alpha^{\prime}}+\varepsilon^{*} \otimes r_{\alpha^{\prime \prime}}$.

It is clear that we have this possibility for each sector $\mathscr{A}_{g}$, i.e. we can tensor with a representation $\varepsilon_{g}$ of $N_{g}$. In principle, the ambiguity could be resolved by demanding the ground state to be invariant. This is an appropriate procedure for the untwisted states. In particular we want $\mathscr{A}_{0}$ to contain the identity operator. For the twisted states however, this is not a good prescription, since the ground state can very well be degenerate, and consequently the prescription would be illdefined. So we will regard the transformation

$$
r_{\alpha}^{g} \rightarrow \varepsilon_{g} \otimes r_{\alpha}^{g}
$$

as a gauge degree of freedom in our description.

What are the possible couplings between these twisted $A_{0}$ representations $\left[\phi_{\alpha}^{A}\right]$, i.e. what is the fusion algebra

$$
\phi_{\alpha}^{A} \times \phi_{\beta}^{B}=\sum_{\gamma, C} N_{\alpha \beta \gamma}^{A B C} \phi_{\gamma}^{C} .
$$

Let us first concentrate on the index labelling the classes of $G$. There are some general rules that govern orbifold interactions (for a discussion of some general aspects of interactions see $[16,17])$. Fix the conjugacy classes $C_{A}, C_{B}$ and $C_{C}$, and consider an interaction between two states in $\mathscr{A}_{A}$ and $\mathscr{A}_{B}$ that gives rise to a state in $\mathscr{A}_{C}$. If we choose $g_{1} \in C_{A}$ and $g_{2} \in C_{B}$ to represent the twisted boundary conditions, then it is clear that the fused state is twisted by an element in the class of $g_{1} g_{2}$. This gives a selection rule on the possible clases $C_{C}$. Given such an allowed triplet of conjugacy classes, how many inequivalent couplings exist? Although the three twists $g_{1}, g_{2}, g_{1} g_{2}$ are only defined up to conjugation, it is not difficult to see that the three point interaction vertex in fact only allows a simultaneous conjugation. Therefore different interaction channels between three classes can occur, corresponding to the inequivalent triplets of representatives of the three conjugacy classes ${ }^{3}$. To be more precise, consider the set

$$
S=\left\{\left(g_{1}, g_{2}, g_{3}\right) \in C_{A} \times C_{B} \times C_{C} \mid g_{1} g_{2}=g_{3}\right\} .
$$

The group $G$ acts on these triplets by simultaneous conjugation of all three elements and under this action we can decompose $S$ into orbits $C^{(i)}$. The fact that the three point coupling is only invariant under simultaneous conjugation implies

\footnotetext{
${ }^{3}$ As an example where there are more than one way to couple three given conjugacy classes, consider the tetrahedron group, and the coupling between two elements in the same conjugacy class with elements of order three, and one in the other conjugacy class with elements of order three. In this case there are two inequivalent couplings possible
} 
that each orbit $C^{(i)}$ corresponds to a different channel between three external states in the sectors $\mathscr{A}_{A}, \mathscr{A}_{B}$, and $\mathscr{A}_{C}$. If we denote by $N^{A B C}$ the number of orbits $C^{(i)}$ that $S$ splits into, we can define the class algebra by

$$
\phi^{A} \times \phi^{B}=\sum_{C} N^{A B C} \phi^{C}
$$

It is not difficult to verify that this algebra is associative and commutative.

This class algebra is of course not yet the complete orbifold fusion algebra (3.14), since we still have to include the representations of the stabilizer subgroups $N_{g i}$. Again, similar as in the untwisted sector, we have to determine the selection rules that follow from the group action on the couplings. It is clear that the three point coupling for a given triplet $\left(g_{1}, g_{2}, g_{1} g_{2}\right) \in C^{(i)}$ cannot be invariant under the full action of the three $N_{g i}$ 's but only under that of the common stabilizer $N^{(i)}=N_{g_{1}} \cap N_{g_{2}}$ acting on $\mathscr{A}_{g_{1}} \otimes \mathscr{A}_{g_{2}} \otimes \mathscr{A}_{g_{1} g_{2}}$. One would now like to repeat the arguments given for the non-twisted sector to construct a correspondence between the possible $\mathscr{A}_{0}$ invariant couplings and the Clebsch-Gordan coefficients of $N^{(i)}$. However, to do this we will have to identify the action of (a subgroup of) $G$ in different Hilbert spaces. As explained above, this action is only well-defined up to a one-dimensional representation. In principle it is possible that when comparing the different representations of $N^{(i)}$ in each of the sectors $\left(g_{1}, g_{2}, g_{1} g_{2}\right)$ we may have to redefine our representation labellings. So, without further information about the details of the orbifold model, the only restriction we can make is that the couplings in $\mathscr{A}_{g_{1}} \otimes \mathscr{A}_{g_{2}} \otimes \mathscr{A}_{g_{1} g_{2}}$ correspond to the $N^{(i)}$-invariant tensors on

$$
r_{\alpha}^{g_{1}} \otimes r_{\beta}^{g_{2}} \otimes r_{\gamma^{*}}^{g_{1} g_{2}} \otimes \varepsilon^{(i)}
$$

where $\varepsilon^{(i)}$ is an a priori arbitrary one-dimensional representation of $N^{(i)}$. Let us denote the number of inequivalent $N^{(i)}$-invariant tensors by $N_{\alpha \beta \gamma}^{(i)}$, that is, we have

$$
r_{\alpha}^{g_{1}} \otimes r_{\beta}^{g_{2}} \otimes r_{\gamma^{*}}^{g_{1} g_{2}} \otimes \varepsilon^{(i)}=N_{\alpha \beta \gamma}^{(i)} 1^{(i)} \otimes \cdots
$$

where $1^{(i)}$ equals the identity representation of $N^{(i)}$. The coefficients $N_{\alpha \beta \gamma}^{A B C}$ of the fusion algebra, finally, are now given by

$$
N_{\alpha \beta \gamma}^{A B C}=\sum_{i=1}^{N A B C} N_{\alpha \beta \gamma}^{(i)},
$$

where the sum is over all orbits $C^{(i)}$ of the set $S$ defined in (3.15). By construction, the number on the right-hand side is the total number of inequivalent couplings in $\left[\phi_{\alpha}^{A}\right] \otimes\left[\phi_{\beta}^{B}\right] \otimes\left[\phi_{\gamma}^{B}\right]$.

Thus we find that the fusion algebra of the holomorphic $G$-orbifold theory naturally combines both the representation and class algebra of the group $G$. From the group theory point of view this is quite interesting, because one knows that the number of conjugacy classes and the number of irreducible representations of a finite group are equal. However there is no canonical correspondence between the two. It is interesting that the orbifolds fuse the two notions into one setting. We return to this point in Sect. 4.c.

This concludes our discussion of the interactions of the general orbifolds of holomorphic conformal theories. It will be clear that the reasoning given in this section has been more intuitive than rigorous. In the next section, however, we will 
provide a more solid base to the arguments presented here, by deriving the same results using the modular transformation of the one-loop characters. There we will further discuss the role of the one-dimensional representations $\varepsilon^{(i)}$ and derive the restrictions it has to satisfy.

\section{Orbifold Models at Genus One}

Much of the structure of a RCFT can be investigated by considering the modular properties of the model on a one-loop Riemann surface. Not only the representation content can be determined [3], but quite unexpectedly also the fusion algebra can be expressed in terms of the representation of the modular group [5]. In this section we will use these powerful results to study the operator algebra of the orbifold CFT's, and to find confirmation of the general arguments presented in the previous section.

\section{4.a. Partition Functions and Characters}

Our starting point will be the path-integral representation of an orbifold partition function $\mathscr{Z}(\tau, \bar{\tau})$ on a torus with modular parameter $\tau$. After one chooses a basis of homology cycles $(a, b)$ on the surface, $\mathscr{Z}$ can be represented as

$$
\mathscr{Z}=\frac{1}{|G|} \sum_{\substack{g, h \in G \\[g, h]=1}} Z(g, h) \varepsilon(g \mid h) .
$$

Here the term $Z(g, h)$ represents the partition function evaluated with boundary conditions twisted by $g$ and $h$ along the $a$ and $b$ cycle respectively. These terms can only be consistently defined if the elements $g$ and $h$ commute. The partition function of the original model, i.e. with periodic boundary conditions, is simply given by $\mathscr{Z}_{0}=Z(1,1)$. In general an explicit evaluation of (4.1) is only possible if a Lagrangian formulation of the model exists. This in particular is the case for toroidal models which are essentially free theories. The phase $\varepsilon(g \mid h)$ that we included is called discrete torsion [18]. We will set $\varepsilon=1$ in the subsequent discussions, but we will return to its interpretation in Sect. 4e.

The expression (4.1) is motivated by the following Hamiltonian interpretation [15], which also accounts for the correct normalization. First the idea of an orbifold clearly implies that we keep only the $G$-invariant states in the original Hilbert space $\mathscr{H}_{0}$. However, in $2 \mathrm{~d}$ field theory we can have non-trivial monodromy, so we also have to include twisted sectors. So more generally, for each element $g \in G$ we can construct a twisted Hilbert space $\mathscr{H}_{g}$ where the fundamental fields obey boundary conditions twisted by $g$. This space of twisted states only depends on the conjugacy class $C_{A}$ of $g$. In each sector we have to project onto the states invariant under the stabilizer group $N_{g}$.

To see that the partition function indeed reflects this structure, we write it as

$$
Z=\sum_{A} \frac{1}{\left|N_{A}\right|} \sum_{h \in N_{A}} Z(g, h), \quad g \in C_{A}
$$

where we used $Z\left(c g c^{-1}, c h c^{-1}\right)=Z(g, h)$ and $\left|N_{A}\right|=|G| /\left|C_{A}\right|$. The operators 


$$
P_{0}^{A}=\frac{1}{\left|N_{A}\right|} \sum_{h \in N_{A}} h
$$

project onto the $N_{A}$-invariant states of $\mathscr{H}_{A}$. So (4.1) can indeed be rewritten as

$$
Z=\sum_{A} \operatorname{Tr}_{\mathscr{H}_{A}} P_{0}^{A} q^{L_{0-c / 24}} \bar{q}^{\bar{L}_{0-c / 24}}
$$

where $q=e^{2 \pi i \tau}$, in accordance with the Hamiltonian picture.

Let us return to the operators $P_{0}^{A}$. We can give a bit more general definition that we will need in a moment. We will denote the irreducible representations of $N_{A}$ as $r_{\alpha}^{A}$ and their characters as $\varrho_{\alpha}^{A}(h)=\operatorname{tr} r_{\alpha}^{A}(h)$. We further define $d_{\alpha}^{A}$ to be the dimension of $r_{\alpha}^{A}$, so that $d_{\alpha}^{A}=\varrho_{\alpha}^{A}(1)$. Now consider the operators $P_{\alpha}^{A}$ that project on the irreducible representation $r_{\alpha}^{A}$,

$$
P_{\alpha}^{A}=\frac{1}{\left|N_{A}\right|} \sum_{h \in N_{A}} \varrho_{\alpha}^{A}(1) \varrho_{\alpha}^{A}\left(h^{-1}\right) h .
$$

They should be considered as elements of the group algebra, or less abstractly as sums of matrices in some representation. They satisfy

$$
\begin{aligned}
r_{\beta}^{A}\left(P_{\alpha}^{A} g\right) & =\delta_{\alpha \beta} r_{\alpha}^{A}(g), \\
\left(P_{\alpha}^{A}\right)^{2} & =P_{\alpha}^{A}, \\
P_{\alpha}^{A} P_{\beta}^{A} & =0, \quad \alpha \neq \beta, \\
\sum_{\alpha} P_{\alpha}^{A} & =1 .
\end{aligned}
$$

It is not difficult to deduce these relations using the general orthogonality relations given in the appendix.

For the case of a holomorphic theory we can now make contact with the description given in Sect. 3. We have complete factorization of the Hilbert space in holomorphic and anti-holomorphic parts,

$$
\mathscr{H}=\mathscr{H}_{0} \otimes \overline{\mathscr{H}}_{0} .
$$

This generalizes to the twisted Hilbert spaces; they are also of the form $\mathscr{H}_{g} \otimes \overline{\mathscr{H}}_{g}$. Furthermore the action of the stabilizers $N_{g}$ decomposes in a left and right action. Accordingly we can define the holomorphic blocks

$$
h \square_{g}=\operatorname{Tr}_{\mathscr{H}_{g}} h q^{L_{0-c / 24}}
$$

and similar expressions for the anti-holomorphic blocks. The partition function $Z(g, h)$ is the product of the holomorphic piece times the anti-holomorphic piece. For a left-right symmetric theory this would be

$$
\left|h \square_{g}\right|^{2}=Z(g, h) \text {. }
$$

As explained in Sect. 3 the holomorphic space $\mathscr{H}_{g}$ decomposes into sectors that transform in an irreducible representation $r_{\alpha}^{g}$ of the stabilizer $N_{g}$ :

$$
\mathscr{H}_{g}=\bigoplus_{\alpha}\left[\phi_{\alpha}^{g}\right] \otimes r_{\alpha}^{g} .
$$


Using the projection operators $P_{\alpha}^{A}$ the one-loop characters $\chi_{\alpha}^{g}(q)$ of the module $\left[\phi_{\alpha}^{g}\right]$ is calculated as follows:

$$
\begin{aligned}
\chi_{\alpha}^{g}(q) & =\operatorname{Tr}_{\left[\phi_{\alpha}^{g}\right]} q^{L_{0-c / 24}}=\frac{1}{d_{\alpha}^{g}} \operatorname{Tr}_{\mathscr{H} g} P_{\alpha}^{g} q^{L_{0}-c / 24} \\
& =\frac{1}{\left|N_{g}\right|} \sum_{h \in N_{g}} \varrho_{\alpha}^{g}\left(h^{-1}\right) h \square_{g}=\frac{1}{|G|} \sum_{\substack{h \in G, g \in C_{A} \\
[h, g]=1}} \varrho_{\alpha}^{g}\left(h^{-1}\right) h \square_{g} .
\end{aligned}
$$

It is clear that these characters $\chi_{\alpha}^{g}(q)$ have a $q$-expansion with positive integer coefficients. The above relation between the characters and the holomorphic blocks can be inverted to give

$$
h \square_{g}=\sum_{\alpha} \varrho_{\alpha}^{g}(h) \chi_{\alpha}^{g}(q) .
$$

Of course the characters depend only on the class $C_{A}$ of $g$. The equations (4.144.15 ) can be considered as a basis transformation in the vector space $V_{1}$ of genus 1 characters.

Using this relation the full partition function can now be written as

$$
Z(q, \bar{q})=\sum_{\alpha, A}\left|\chi_{\alpha}^{A}(q)\right|^{2}
$$

(where again for simplicity of notation we have assumed left-right symmetric models). We note that the ambiguity (3.13) in the labelling of the operators corresponds in terms of the chiral blocks to a redefinition

$$
h \square_{g} \rightarrow \varepsilon_{g}(h) h \underset{g}{\square}
$$

with $\varepsilon_{g}$ a one-dimensional representation of $N_{g}$. A transformation of type (4.17) is always accompanied by a similar transformation with $\varepsilon_{g}^{*}$ acting on the antiholomorphic blocks. In this way the modular invariance of the partition function is preserved.

\section{4.b. Modular Transformations}

Quite generally a RCFT defined on an arbitrary Riemann surface obtains a unitary representation of the modular group on its generalized characters. This can be seen as a direct consequence of the fundamental assumption of modular invariance [21,22,9]. For the non-chiral blocks $Z(g, h)$ in genus one, we have a simple transformation rule under the modular group. Recall that modular transformations act on the homology basis $(a, b)$ as $S L(2, \mathbf{Z})$ transformations. This gives a straightforward action on the boundary conditions used to define $Z(g, h)$ :

$$
M=\left(\begin{array}{ll}
\alpha & \beta \\
\gamma & \delta
\end{array}\right) \in S L(2, \mathbf{Z}): Z(g, h) \rightarrow Z\left(g^{\alpha} h^{\beta}, g^{\gamma} h^{\delta}\right) .
$$

For the chiral blocks however, which are the holomorphic square roots of the twisted partition function $Z(g, h)$, this transformation rule is somewhat modified, since in general a phase has to be included ${ }^{4}$

\footnotetext{
${ }^{4}$ We would like to thank L. Dixon for a discussion on the importance of these phases
} 


$$
M: h \square_{g} \rightarrow \varepsilon_{M}(g \mid h) g^{\gamma} h^{\delta} \square_{g^{\alpha} h^{\beta}},
$$

where $\varepsilon_{M}$ is a phase factor independent of $\tau$. Since the anti-chiral blocks transform with the opposite phases, it is evident that in the partition function the two phases will cancel ${ }^{5}$. In Sect. 5 we will show that for generic models these phases indeed occur. They will play an important part in the subsequent analysis, and we will treat them in detail.

As is well-known, the modular group is generated by the transformation $S$ and $T$, defined on the cycles as:

$$
\begin{aligned}
& S:(a, b) \rightarrow(b,-a), \\
& T:(a, b) \rightarrow(a, a+b),
\end{aligned}
$$

with the relations $S^{2}=(S T)^{3}=\mathscr{C}$, where the charge conjugation reverses the orientation of the cycles. We will first consider $S$, which interchanges the "space" and "time" directions. Among other things, $S$ will give us the fusion rules of the model. Let us now define the phases $\sigma(g \mid h)$ by

$$
S: h \square_{g} \rightarrow \sigma(g \mid h) g^{-1} \square_{h} .
$$

We will derive the following consistency conditions on $\sigma(g \mid h)$ :

$$
\begin{gathered}
\sigma(h \mid g)=\sigma(g \mid h), \\
\delta_{1} \delta_{2} \sigma\left(h_{1}, h_{2} \mid g_{1}, g_{2}\right)=1 .
\end{gathered}
$$

In the last condition we introduced the coboundary operators $\delta_{1}, \delta_{2}$. They act as the coboundary operator $\delta$ of group cohomology on the two arguments of $\sigma$ :

$$
\begin{aligned}
& \delta_{1} \sigma\left(h_{1}, h_{2} \mid g\right)=\sigma\left(h_{1} \mid g\right) \sigma\left(h_{2} \mid g\right) \sigma^{-1}\left(h_{1} h_{2} \mid g\right), \\
& \delta_{2} \sigma\left(h \mid g_{1}, g_{2}\right)=\sigma\left(h \mid g_{1}\right) \sigma\left(h \mid g_{2}\right) \sigma^{-1}\left(h \mid g_{1} g_{2}\right) .
\end{aligned}
$$

Note that $\delta_{1} \delta_{2}=\delta_{2} \delta_{1} \cdot \varepsilon_{2}$ the condition $\delta_{1} \sigma=1$ implies that $\sigma(h \mid g)$, when considered as a function of $h$, is a one-dimensional representation of $N_{g}$. Consequently $\delta_{1} \delta_{2} \sigma=1$ has the following interpretation: $\delta_{2} \sigma\left(h \mid g_{1}, g_{2}\right)$ is a 2-coboundary on $N_{h}$ and a representation of $N_{g_{1}} \cap N_{g_{2}}$. We will motivate this condition on $\sigma$ when we discuss the fusion algebra.

The requirement of symmetry (4.23) can be proved as follows. First we observe that the chiral blocks are functions of $\tau$. This implies that the transformation $S$ is implemented as the $P S L(2, \mathbf{Z})$ transformation $\bar{S}: \tau \rightarrow-1 / \tau$, which satisfies $\bar{S}^{2}=1$. Furthermore, if we take $\tau$ pure imaginary, the one-loop characters $\chi_{\alpha}^{g}(\tau)$ are real. Consequently we have in that case the identity

$$
h \square_{g}=\left(h^{-1} \square_{g}\right)^{*} \text {. }
$$

(This is a direct result of $\varrho_{\alpha}^{A}\left(h^{-1}\right)=\varrho_{\alpha}^{A}(h)^{*}$ and relation (4.15).) If we now use this relation and $\bar{S}^{2}=1$ we obtain the result $\sigma(g \mid h)=\sigma(h \mid g)$.

\footnotetext{
${ }^{5}$ In asymmetric orbifolds [23] this cancellation is not automatic and has to be imposed by the socalled level-matching conditions
} 
A further important property of $\sigma(g \mid h)$ is its relation with the charge conjugation operation. Charge conjugation $\mathscr{C}$ clearly leaves the characters invariant, but can reshuffle the indices if several characters have the same $q$ expansion. This implies that in general we can have

$$
\mathscr{C}: h \square_{g} \rightarrow c_{g}(h) h^{-1} \underset{g^{-1}}{\square}
$$

Here $c_{g}(h)$ is a one-dimensional representation of $N_{g}$. By CPT-invariance we have $S^{2}=\mathscr{C}$, so that

which implies

$$
\sigma(g \mid h) \sigma\left(h \mid g^{-1}\right)=c_{g}(h)
$$

$$
c_{g}(h)=c_{g-1}(h) \text {. }
$$

This guarantees the relation $S^{4}=1$. If $g$ and $g^{-1}$ are not conjugate we can remove the phase $c_{g}$ by a gauge transformation (4.17). Finally, note that all conditions we imposed on the phase factors are invariant under the transformation (4.17), since it implies

$$
\sigma(g \mid h) \rightarrow \varepsilon_{g}(h) \varepsilon_{h}(g) \sigma(g \mid h)
$$

which can be readily seen not to interfere with the above conditions.

We now proceed to give the action of $S$ in the basis of one-loop characters $\chi_{\alpha}^{A}$ instead of chiral blocks:

$$
\begin{aligned}
S: \chi_{\alpha}^{A} & \rightarrow \frac{1}{|G|} \sum_{\substack{\left.h \in G, g \in C_{A} \\
h, g\right]=1}} \varrho_{\alpha}^{g}\left(h^{-1}\right) \sigma(g \mid h) g^{-1} \square \\
& =\frac{1}{|G|} \sum_{\substack { \beta, B \\
\begin{subarray}{c}{h \in C_{B}, g \in C_{A} \\
[h, g]=1{ \beta , B \\
\begin{subarray} { c } { h \in C _ { B } , g \in C _ { A } \\
[ h , g ] = 1 } }\end{subarray}} \varrho_{\alpha}^{g}\left(h^{-1}\right) \varrho_{\beta}^{h}\left(g^{-1}\right) \sigma(g \mid h) \chi_{\beta}^{B} .
\end{aligned}
$$

This gives the following elegant expression for the matrix elements of $S$ :

$$
S_{\alpha \beta}^{A B}=\frac{1}{|G|} \sum_{\substack{h \in C_{B}, g \in C_{A} \\[h, g]=1}} \varrho_{\alpha}^{g}\left(h^{-1}\right) \varrho_{\beta}^{h}\left(g^{-1}\right) \sigma(g \mid h) .
$$

From general arguments we know that $S$ should be symmetric and unitary. The first property is ensured by condition (4.23), while unitarity follows from the fact that $S^{*}=S^{-1}$.

We will now use the modular transformation $T$ to derive the conformal weights of the twist fields in terms of the $U(1)$-cocycle $\sigma$. It is clear that, similar as for the transformation $S$, we have to include an (a priori arbitrary) phase factor $\tau(g \mid h)$ in the transformation rules of $T$,

$$
T: h \square_{g} \rightarrow e^{-2 \pi i c / 24} \tau(g \mid h) h g \square_{g} .
$$

Here we included the factor $e^{-2 \pi i c / 24}$ for convenience. Since $T$ should be diagonal in the basis $\chi_{\alpha}^{A}$, we immediately see that $\tau(g \mid h)=\tau_{g}$. Furthermore $\tau_{g}$ only depends on the conjugacy class $C_{A}$ of $g$. Note that

$$
\tau_{g}=e^{2 \pi i h_{g}}
$$


where $h_{g}$ is the ground state energy of $\mathscr{H}_{g}$. We now apply the fundamental relation $(S T)^{3}=\mathscr{C}$ on a chiral block. This gives us

$$
\tau_{g} \tau_{h} \tau_{g h} \sigma(g \mid h g) \sigma(h \mid g h) \sigma(g \mid h)^{-1}=1 .
$$

Choosing $h=1$ we immediately find a relation between $\tau_{g}$, i.e. the conformal weights, and $\sigma$ :

$$
\tau_{g}^{2}=\sigma(g \mid g)^{-1}
$$

Note that substituting this in (4.37) gives a condition that can be written very succinctly as

$$
\left(\delta_{1} \delta_{2} \sigma\right)(g, h \mid g, h)=1,
$$

which is indeed a special case of the condition (4.24). Consequently the relation $(S T)^{3}=\mathscr{C}$ does not give any further relations on $\sigma$.

It is now not difficult to determine the weights of the other operators. Since $g$ obviously is an element of the center of $N_{g}$, we can use Schur's lemma to show that in any irreducible representation $r_{\alpha}^{g}$ of $N_{g}$ it is represented as a phase factor $\varepsilon_{\alpha}^{g}$ (satisfying $\left(\varepsilon_{\alpha}^{g}\right)^{n}=1$ with $n$ the order of $g$ ) times the identity

$$
r_{\alpha}^{g}(g)=\varepsilon_{\alpha}^{g} 1
$$

From this we find that $T$ acts on the character of the chiral sector $\left[\phi_{\alpha}^{g}\right]$ as

$$
\begin{aligned}
T: \chi_{\alpha}^{g} & \rightarrow e^{-2 \pi i c / 24} \tau_{g} \frac{1}{\left|N_{g}\right|} \sum_{h \in N_{g}} \varrho_{\alpha}^{g}\left(h^{-1}\right) h g \underset{g}{\square} \\
& =e^{-2 \pi i c / 24} \tau_{g} \frac{1}{\left|N_{g}\right|} \sum_{h \in N_{g}} \varrho_{\alpha}^{g}\left(g h^{-1}\right) h \square_{g} \\
& =e^{-2 \pi i c / 24} \tau_{g} \varepsilon_{\alpha}^{g} \chi_{\alpha}^{g} .
\end{aligned}
$$

Hence we obtain the important result that all operators in $\left[\phi_{\alpha}^{g}\right]$ have conformal weight given by

$$
e^{2 \pi i h_{g, \alpha}}=\tau_{g} \varepsilon_{\alpha}^{g}
$$

\section{4.c. The Fusion Algebra}

It is a fundamental result that the fusion algebra of a RCFT can be derived from the transformation properties of the modular group [5]. More explicitly we have the relation

$$
N_{i j k}=\sum_{n} \frac{S_{i n} S_{j n} S_{k n}}{S_{0 n}}
$$

We will now use this result to establish the fusion algebra in the orbifold models

$$
\phi_{\alpha}^{A} \times \phi_{\beta}^{B}=\sum_{\gamma, C} N_{\alpha \beta \gamma}^{A B C} \phi_{\gamma}^{C} .
$$

Since we have explicitly calculated $S(4.45)$ can be easily applied. In order to simplify the resulting expression we need the following result in group theory [24]. Suppose we are given a function $f\left(g_{1}, g_{2}, g_{3}\right)$ that only depends on the classes of its arguments. Then the following identity holds: 


$$
\sum_{g_{\imath} \in G} \frac{1}{|G|} \sum_{\alpha} \frac{1}{d_{\alpha}} \varrho_{\alpha}\left(g_{1}\right) \varrho_{\alpha}\left(g_{2}\right) \varrho_{\alpha}\left(g_{3}^{-1}\right) f\left(g_{1}, g_{2}, g_{3}\right)=\sum_{\substack{g_{\imath} \in G \\ g_{1} g_{2}=g_{3}}} f\left(g_{1}, g_{2}, g_{3}\right) \text {. }
$$

With this result and after some algebra $N_{\alpha \beta \gamma}^{A B C}$ can be rewritten as

$$
\begin{aligned}
N_{\alpha \beta \gamma}^{A B C} & =\frac{1}{|G|} \sum_{\substack{g_{1} \in C_{A}, g_{2} \in C_{B}, g_{3} \in C_{C}, h \in G \\
\left[h, g_{1}\right]=\left[h, g_{2}=\left[h, g_{3}\right]=1 \\
g_{1} g_{2}=g_{3}\right.}} \varrho_{\alpha}^{g_{1}}(h) \varrho_{\beta}^{g_{2}}(h) \varrho_{\gamma}^{g_{3}}\left(h^{-1}\right) \delta_{2} \sigma\left(h \mid g_{1}, g_{2}\right) \\
& =\sum_{i} \frac{1}{\left|N^{(i)}\right|} \sum_{h \in N^{(1)}} \varrho_{\alpha}^{g_{1}}(h) \varrho_{\beta}^{g_{2}}(h) \varrho_{\gamma^{*}}^{g_{1} g_{2}}(h) \delta_{2} \sigma\left(h \mid g_{1}, g_{2}\right) .
\end{aligned}
$$

Here in the second line we can indeed recognize the sum over the different interaction channels, as argued in Sect. 3 (see Eqs. (3.18-3.19)). Furthermore we can now calculate the phase $\varepsilon^{(i)}$. It is given by

$$
\varepsilon^{(i)}(h)=\delta_{2} \sigma\left(h \mid g_{1}, g_{2}\right),
$$

where the label $i$ corresponds to the interaction channel $\left(g_{1}, g_{2}, g_{1} g_{2}\right)$. This relation finally gives our motivation for the condition (4.24) on $\sigma$, since it implies that $\delta_{2} \sigma$ should be a representation of $N^{(i)}$, i.e. $\delta_{1} \delta_{2} \sigma=1$.

The determination of the operator algebra of the conformal field theories that can be constructed as a $G$-orbifold of a holomorphic theory is (at least as far as their fusion rules are concerned) now reduced to a cohomology problem. They are labelled by the solutions to the conditions solutions of $\delta_{1} \delta_{2} \sigma=1$ modulo the pure gauge solutions $\sigma(g \mid h)=\varepsilon_{g}(h) \varepsilon_{h}(g)$. It would be interesting to try to derive these conditions more directly, without appealing to the relation between the fusion algebra and the modular transformation matrix, perhaps by using conditions of modular invariance at higher loops.

The above result for the interaction rules and the phase $\sigma$ can also be given the following interpretation. We can redefine the action $r_{g}(h)$ of $N_{g}$ in the sector $\mathscr{H}_{g}$ by

$$
r_{g}(h) \rightarrow r_{g}^{\prime}(h)=\sigma(h \mid g) r_{g}(h) .
$$

The new representation $r_{g}^{\prime}$ has a trivial 2-cocycle

with

$$
r_{g}^{\prime}\left(h_{1}\right) r_{g}^{\prime}\left(h_{2}\right)=c\left(h_{1}, h_{2} \mid g\right) r_{g}^{\prime}\left(h_{1} h_{2}\right)
$$

$$
c\left(h_{1}, h_{2} \mid g\right)=\delta_{1} \sigma\left(h_{1}, h_{2} \mid g\right) .
$$

With the use of these representations $r_{g}^{\prime}$ the fusion rules can now be interpreted straightforwardly as the couplings invariant under the mutual stabilizer $N^{(i)}$, i.e. without the phase ambiguity $\varepsilon^{(i)}$. How can we understand the relation $\delta_{1} \delta_{2} \sigma=1$ from this point of view? Let us introduce operators $\Phi_{g}$ that create sectors twisted by $g$ in the non-local model. Now consider the operator $\Phi_{g_{1}} \Phi_{g_{2}} \Phi_{\left(g_{1} g_{2}\right)^{-1}}$. We claim that this operator is an element of the chiral algebra, and should accordingly transform in a true representation. This implies the following condition on the cocycles

$$
\delta_{2} c=\delta_{2} \delta_{1} \sigma=1
$$


So we have two ways of interpreting the phase $\sigma$ in an operator formulation. Either we insist on having true representations of the stabilizers, in which case $\sigma$ enters via the phase $\varepsilon^{(i)}=\delta_{2} \sigma$ relating the representations of $N^{(i)}$ in the different chiral sectors, or we demand that there are no phases in the interaction rule and instead allow for representations with trivial cocycles $c=\delta_{1} \sigma$. The two different interpretations are related essentially by the modular transformation $S$.

We will now briefly discuss the modifications for the case when the action of the stabilizer subgroups $N_{g}$ in the twisted sectors have non-trivial cocycles $c\left(h_{1}, h_{2} \mid g\right)$. We should note, however, that no examples of orbifolds with this structure are known to us. In particular the orbifolds based on tori seem to give rise to trivial cocycles, as the stabilizer group is actually representable on the twisted sectors. But since we do not have a general argument why they cannot appear for an arbitrary conformal theory, we will continue with their study. The non-triviality of the cocycles means that it is not possible to eliminate them by redefining the action of the stabilizers. Using modular invariance, however, this can be seen to imply that it is also impossible to give a straightforward operator interpretation of the interaction rules, i.e. there also exists an obstruction to eliminate the phase ambiguity $\varepsilon^{(i)}$. Indeed, it can be shown that the phase $\varepsilon^{(i)}$ is no longer given by (4.50), but receives a contribution of the cocycle

$$
\varepsilon^{(i)}(h)=c\left(g_{1}, g_{2} \mid h\right) \delta_{2} \sigma\left(h \mid g_{1}, g_{2}\right) .
$$

The fusion rules are still given by an expression of the form (4.49), but with $\delta_{2} \sigma$ replaced by $c \delta_{2} \sigma$. Finally, the condition (4.24) on $\sigma$ is modified to

$$
\delta_{1} \delta_{2} \sigma \delta_{1} c \delta_{2} c=1
$$

which follows from the fact that $\varepsilon^{(i)}$ has to be a one-dimensional representation of $N^{(i)}$ with cocycle $\delta_{2} c^{-1}$.

Let us make some further remarks on the fusion algebra. In general the irreducible representations of the fusion algebra, which are one-dimensional since the algebra is commutative and associative, are given by $\lambda_{i}^{(j)}=S_{i j} / S_{0 j}$ [5]. This equation implies a canonical identification between the elements of the algebra and its representations. This is a hint of a deeper kind of self-duality inherent to conformal field theory. For the orbifold models this can be made more explicit. Consider the one-dimensional representations of the fusion algebra $\lambda_{\alpha, A}^{(\beta, B)}$. They form an extension of the character table of a finite group to a self-dual object. In particular we have $\lambda_{\alpha, 0}^{(0, B)}=\varrho_{\alpha}\left(C_{B}\right)$ and $\lambda_{\alpha, A}^{(0, B)}=\varrho_{\alpha^{\prime}}\left(C_{B}\right)$ with $r_{\alpha^{\prime}}$ the representation of $G$ obtained by extending the representation of $N_{A}$ to all of $G$ (the so-called induced representation [24]). The dual object of a character table is the collection $\left\{\left|C_{A}\right| \varrho_{\alpha}\left(C_{A}\right) / d_{\alpha}\right\}$ that satisfy the class algebra, and are given by the $\lambda_{0,0}^{\alpha, 0}$.

The fusion algebra for the case $\sigma=1$ also appears in the mathematical literature, in particular in the work of Lusztig [25], where it is developed in the study of Lie groups over a finite field. The appropriate objects in that context are $G$-equivariant complex vector bundles over the group $G$. Since $G$ is a finite set, an equivariant vector bundle over $G$ is simply a collection of finite vector spaces $V_{g}$ with a representation of $G$ on

$$
V=\bigoplus_{g \in G} V_{g}
$$


such that $g V_{h}=V_{g h g^{-1}}$. The set of all these equivariant bundles with the obvious notion of addition is called the Grothendieck group $K_{G}(G)$. The irreducible vector bundles, i.e. bundles $V$ that cannot be written as $V_{1} \oplus V_{2}$, are labelled by a conjugacy class $C_{A}$ and an irreducible representation of the stabilizer subgroup $N_{A}$. These bundles satisfy $V_{g} \neq 0$ if and only if $g \in C_{A}$ and carry the irreducible representation $r_{\alpha}^{g}$ of $N_{g}$. There is also a definition of multiplication (which is not the tensor product) which makes $K_{G}(G)$ into a semisimple commutative algebra. The definition is [25]

$$
\left(V \cdot V^{\prime}\right)_{g}=\bigoplus_{\substack{g_{1}, g_{2} \in G \\ g_{1} g_{2}=g}}\left(V_{g_{1}} \otimes V_{g_{2}}^{\prime}\right)
$$

It is not difficult to see that we can identify the Grothendieck algebra $K_{G}(G)$ with our fusion algebra for the case $\sigma=1$. The matrix $S$ is also featured in the work of Lusztig where it is called a non-abelian Fourier transformation.

As explained in [10] for a general RCFT the quantities $\lambda_{0}^{(i)}=S_{0 i} / S_{00}$ indicate the relative dimension $\left[\phi_{i}: \phi_{0}\right]$. More precisely, we define the relative dimension of a module $\left[\phi_{i}\right]$ with respect to the basic module $\left[\phi_{0}\right]$ by the following limit:

$$
\left[\phi_{i}: \phi_{0}\right]=\lim _{q \rightarrow 1} \frac{\chi_{i}(q)}{\chi_{0}(q)}
$$

If we apply the modular transformation $S$ and suppose unitarity, this can be evaluated to give $\left[\phi_{i}: \phi_{0}\right]=\lambda_{i}^{(0)}$. In general this will not be an integer. In the case of the orbifold models here at hand we find

$$
\left[\phi_{\alpha}^{A}: \phi_{0}\right]=d_{\alpha}^{A}\left|C_{A}\right| \text {. }
$$

This follows also immediately from the relation with equivariant bundles over $G$. The irreducible vector bundles clearly have dimension $d_{\alpha}^{A}\left|C_{A}\right|$. These relative dimensions further equal the dimensions of the induced representations of $G$. The above relation is a somewhat puzzling result: although we modded out the group $G$ and do not have an action of it anymore, the relative dimension still equals the dimension of the representation of the finite group. In particular we can calculate

using $\sum_{\alpha} d_{\alpha}^{2}=|G|$ (see appendix).

$$
\left[\mathscr{A}: \mathscr{A}_{0}\right]=\sum_{\alpha} d_{\alpha}\left[\phi_{\alpha}: \phi_{0}\right]=|G|
$$

\section{4.d. Higher Genus Riemann Surfaces}

The partition function of a RCFT on a Riemann surface of genus $g$ described by moduli $\left(m_{\alpha}, \bar{m}_{\alpha}\right)(\alpha=1, \ldots, 3 g-3)$ will again be a sum of terms that factorize inholomorphic times anti-holomorphic functions of the moduli $m_{\alpha}$. One way to understand this analytic structure is by constructing a Riemann surface from sewing thrice-punctured spheres and projecting on modules $\left[\phi_{i}\right] \otimes\left[\bar{\phi}_{\bar{i}}\right]$ in the intermediate channels. Analyticity is then guaranteed by construction. The resulting generalized characters are holomorphic sections of the so-called Friedan-Shenker vector bundle over the moduli space of Riemann surfaces [21].

In the case of an orbifold of an holomorphic theory, we have another natural basis of this bundle, viz. the twisted sectors. Given a canonical homology basis 
$\left(a_{i}, b_{i}\right)(i=1, \ldots, g)$ we can have twists $g\left(a_{i}\right), g\left(b_{i}\right) \in G$ along all the cycles, where the group elements are again defined up to simultaneous conjugation. They have to satisfy

$$
\prod_{i}\left[g\left(a_{i}\right), g\left(b_{i}\right)\right]=1 .
$$

This is the generalization of the one-loop constraint $[g(a), g(b)]=1$. The set $g\left(a_{i}\right)$, $g\left(b_{i}\right)$ defines an homomorphism from the fundamental group of the Riemann surface to the group $G$. As such it defines a $G$ bundle over the surface [26]. Two such homomorphisms give equivalent bundles if they are related by a global conjugation of a group element. The chiral twisted sectors are defined by taking an holomorphic square root. That it is possible to take this square root can be seen by considering the theory on a covering of the Riemann surface. The partition function can be expressed as a sum of the modulus squared of the chiral twisted sectors, or a similar expression using the holomorphic blocks. This implies that there will be a linear basis transformation that expresses the twisted sectors into the generalized characters.

As explained in [5] one can calculate the number of generalized characters on a Riemann surface of genus $g$ using the matrix elements $S_{0 i}$. This number equals the dimension of the Friedan-Shenker bundle $V_{g}$ over the moduli space of $g$-loop surfaces. The result reads

$$
\operatorname{dim} V_{g}=\sum_{i}\left|S_{0 i}\right|^{2(1-g)}
$$

This number counts the number of ways a $\varphi^{3}$-diagram can be "colored," taking into account the fusion rules.

If we apply this formula to the case of an orbifold model of an holomorphic theory, we also obtain the number of independent twisted sectors. Mathematically this corresponds to the number of inequivalent $G$-bundles over the Riemann surface. To our knowledge an explicit expression for this quantity was not yet known. Substituting (4.34) in (4.63) we find

$$
\operatorname{dim} V_{g}=\sum_{A, \alpha}\left[\frac{N_{A}}{d_{\alpha}^{A}}\right]^{2(g-1)} .
$$

This is indeed always an integer, since it is a fundamental result in the theory of finite groups that the dimension of an irreducible representation always divides the dimension of the group [24]. One can further check that the dimension is indeed one on the sphere with the aid of the relation $\Sigma_{\alpha} d_{\alpha}^{2}=|G|$. For abelian groups the above equation correctly reproduces

$$
\operatorname{dim} V_{g}=|G|^{2 g} .
$$

Note that the dimensions of the Friedan-Shenker bundles do not depend on the particular $\sigma$ used to define the fusion algebra.

Similarly one can easily derive expressions for the dimension of $G$-bundles on a Riemann surface with $n$ punctures (or boundaries) using the appropriate generalization of (4.63) to include external lines. 


\section{4.e. Discrete Torsion}

Up to now we have been concerned with the chiral structure. We considered the algebras $\mathscr{A} / G$, the representations and their modular properties. Now we want to investigate the combination of the holomorphic and anti-holomorphic characters into a modular invariant partition function. Although in principle we can choose different left and right chiral algebra, we will restrict ourselves here to the symmetric case.

Different modular invariant partition functions are classified by constructing all possible permutations $\pi$ of the space of one-loop characters that commute with the modular group. As shown in $[10,11]$ this implies that $\pi$ also constitutes an automorphism of the fusion algebra. One way to obtain different modular invariant partition functions for orbifold models can be done by the inclusion of a discrete torsion $\varepsilon(g \mid h)$ in the expression for the partition function [18]. We will now show that both approaches are equivalent.

Let us recall the conditions that the phase $\varepsilon(g \mid h)$ has to satisfy

$$
\begin{gathered}
\delta_{1} \varepsilon\left(h_{1}, h_{2} \mid g\right)=1, \\
\varepsilon(h \mid g)=\varepsilon\left(g^{-1} \mid h\right), \\
\varepsilon(g \mid g)=1 .
\end{gathered}
$$

The relation $\delta_{1} \varepsilon=1 \mathrm{implies}$ that $\varepsilon(h \mid g)$ is a one-dimensional representation of $N_{h}$. The symmetry property $\varepsilon(h \mid g)=\varepsilon\left(g^{-1} \mid h\right)$ makes it also a representation of $N_{g}$. This implies that we can define an action of $\varepsilon_{g}(h)=\varepsilon(g \mid h)$ on the one-loop characters by permuting the representations of $N_{g}$, completely similar to the action (3.13),

$$
\pi_{\varepsilon}: \chi_{\alpha}^{g} \rightarrow \chi_{\varepsilon_{g} \otimes \alpha}^{g} .
$$

However, here it acts only in the holomorphic characters, while leaving the antiholomorphic ones invariant, and accordingly the partition function will not be invariant. In fact we have constructed a permutation of the characters. We now have to show that $\pi_{\varepsilon}$ commutes with the modular transformations: $\pi_{\varepsilon} S=S \pi_{\varepsilon}$ and $\pi_{\varepsilon} T=T \pi_{\varepsilon}$. Both are easy results of the conditions imposed on $\varepsilon$.

\section{Examples}

In this section we will illustrate the structure of orbifolds constructed from holomorphic theories with some concrete examples.

\section{5.a. Abelian Groups and Toroidal Models}

Many of the results become more transparent when we consider an abelian group $G$. In that case the twisted sectors are labelled by the elements of $G$, and contain all irreducible representations. So the total number of primary operators is $|G|^{2}$. Furthermore the class algebra and the representation algebra are simply isomorphic to $G$. Without the inclusion of the phases $\sigma$ these two algebras would completely decouple. This would result in a fusion algebra given by (the group algebra) of $G \times G$. However, the occurrence of the phases is a generic pheno- 
menon, as can be shown by considering the toroidal compactifications. These examples will also help to clarify the relation between the phases in the modular transformations and the resulting modification of the fusion algebra.

A well-known class of holomorphic theories is given by certain special toroidal compactifications (see [27] and e.g. [20]). These models are described in terms of a free scalar field $\phi^{\mu}(\mu=1, \ldots, d)$ compactified on a torus $\mathbf{R}^{d} / 2 \pi \Lambda$, where $\Lambda$ is an Euclidean even, self-dual lattice, i.e. for all $p, p^{\prime} \in \Lambda$ we have $p^{2} \in 2 \mathbf{Z}$ and $p \cdot p^{\prime} \in \mathbf{Z}$. (This requires of course $d=0 \bmod 8$.) With the inclusion of an appropriate constant antisymmetric background field in the action the partition function is easily evaluated and seen to factorize as

$$
Z(q, \bar{q})=\left|\frac{1}{\eta(q)^{d}} \sum_{p \in \Lambda} q^{\frac{1}{2} p^{2}}\right|^{2} .
$$

We can now construct the following class of abelian orbifolds from these models: We choose a lattice $\Lambda^{\prime} \supset \Lambda$ and mod out by the transformations

$$
\phi \rightarrow \phi+2 \pi \alpha, \quad \alpha \in \Lambda^{\prime} / \Lambda \text {. }
$$

Here the relevant group $G$ is isomorphic to $\Lambda^{\prime} / \Lambda$ considered as an additively written abelian group. The chiral action of the shifts $\alpha$ is easily written down. It only depends on the chiral momentum $p \in \Lambda$ and is given by

$$
\alpha:|p\rangle \rightarrow e^{2 \pi i p \cdot \alpha}|p\rangle \text {. }
$$

Furthermore, it is not difficult to construct the twisted Hilbert spaces $\mathscr{H}_{\alpha}$. They consist of momentum states $|p+\alpha\rangle, p \in \Lambda$. However, the action of $\beta \in G$ on $\mathscr{H}_{\alpha}$ is seen to be ambiguous. It depends on the choice of the representatives $\alpha, \beta \in \Lambda^{\prime}$. This will be reflected in a phase ambiguity in the definition of the chiral blocks. Part of that ambiguity is resolved once we demand that the action of $G$ is linearly, i.e. nonprojectively, represented. If we furthermore choose a set of representatives in $\Lambda^{\prime}$, for example by taking $\alpha^{2}$ minimal (although this can be ambiguous), we obtain a definite expression for the chiral blocks:

$$
\beta \square_{\alpha}=\frac{1}{\eta^{d}} \sum_{p \in \Lambda} e^{2 \pi i\left[\frac{1}{2}(p+\alpha)^{2} \tau+p \cdot \beta\right]} .
$$

The above definition does not depend on the choice $\beta \in \Lambda^{\prime}$,

$$
(\beta+k) \square_{\alpha}=\beta \square_{\alpha}, \quad \forall k \in \Lambda .
$$

This is however no longer true for $\alpha$, since

$$
\beta \underset{\alpha+k}{\square}=e^{-2 \pi i \beta \cdot k} \beta \square_{\alpha}
$$

That is to say, we have been forced to break the symmetry in the cycles $a$ and $b$ by defining time to flow along the $b$-cycle. That is of course in complete accordance with the Hamiltonian point of view. We note that the ambiguity (5.6) is of the form (4.17), i.e. it corresponds to multiplication by a one-dimensional representation of $G$. This definition of the chiral blocks gives rise to a modified action of the modular group. Indeed it is easy to verify that

$$
S: \beta \square_{\alpha} \rightarrow e^{-2 \pi i \alpha \cdot \beta}-\alpha \square_{\beta} \text {. }
$$


So we find for the phase $\sigma$ :

$$
\sigma(\alpha, \beta)=e^{-2 \pi i \alpha \cdot \beta} .
$$

It is not difficult to check that it indeed satisfies all the conditions we imposed. Further note that $\tau(\alpha)^{2}=e^{2 \pi i \alpha^{2}}$ gives the correct weight $h=\frac{1}{2} \alpha^{2}$.

Let us now calculate the characters of the orbifold model. The representations of $G$ can be labelled by elements $\mu \in \Lambda / \Lambda^{\prime *}$, with $\Lambda^{\prime *}$ the dual lattice of $\Lambda^{\prime}$. This results in the following expression for the characters

$$
\chi_{\mu}^{\alpha}=\frac{1}{|G|} \sum_{\beta \in A^{\prime} / \Lambda} e^{-2 \pi i \mu \cdot \beta} \beta \square_{\alpha},
$$

which can be rewritten as

$$
\chi_{\mu}^{\alpha}(q)=\frac{1}{\eta(q)^{d}} \sum_{p \in \Lambda^{\prime *}} q^{\frac{1}{2}(p+\alpha+\mu)^{2}},
$$

an evident result, since we have in fact constructed-in a complicated way-the toroidal model based on the lattice $\Lambda^{\prime}$. It is now also evident what the fusion algebra is. It is given by $\Lambda^{\prime} / \Lambda^{\prime} *$ which is in general not equal to $\Lambda^{\prime} / \Lambda \times \Lambda^{\prime} / \Lambda$. The projective action of $\beta \in G$ on the twisted sectors $\mathscr{H}_{\alpha}$ that accounts correctly for these fusion rules is according to (4.51) given by

$$
\beta:|p\rangle \rightarrow e^{2 \pi i p \cdot \beta}|p\rangle \quad p \in \Lambda+\alpha .
$$

This is indeed the action of a chiral shift $\phi \rightarrow \phi+2 \pi \beta$.

After this example it is not too difficult to treat the general abelian case. For simplicity we restrict ourselves here to $G=\mathbf{Z}_{N}$. Motivated by the above example we write $\mathbf{Z}_{N}$ additively. With an appropriate gauge transformation the phase $\sigma$ can always be given in the following form:

$$
\sigma(\alpha, \beta)=e^{2 \pi i \alpha \beta k / N^{2}},
$$

with $\alpha, \beta \in \mathbf{Z}_{N} \equiv \mathbf{Z} / N \mathbf{Z}$. The conditions (4.23), (4.24) and (4.29) restrict $k \in \mathbf{Z}$. With the inclusion of this expression for $\sigma(\alpha, \beta)$ the fusion algebra equals $\mathbf{Z}_{p N} \times \mathbf{Z}_{N / p}$ with $p=N /(k, N)$. For the case that $N$ is even, there is a further restriction on $\sigma$ which forces $k$ to be even. This is because when we look at the element $g$ of order two in $\mathbf{Z}_{N}$, since $g^{-1}=g$, when applying $S^{2}$ to the one loop character corresponding to twistings by $(g, g)$ in the $(a, b)$ directions, we should get no extra phases. This implies that $\sigma(g \mid g)^{2}=1$, which in turn implies that $k$ is even. To compare with the toroidal case, consider modding $\Lambda$ by a shift vector $v$, with $N v \in \Lambda$. Then if $(N v)^{2}=k \bmod N$, then it is easy to convince oneself that the operator algebra is $\mathbf{Z}_{p N} \times \mathbf{Z}_{N / p}$ with $p=N /(k, N)$. It is clear that because $\Lambda$ is an even lattice, when $N$ is even $k$ is also even, in accordance with the general arguments just discussed (for $N$ odd, since $k$ is only defined modulo addition by $N$ we can take $k$ to be even or odd).

\section{5.b. A Non-Abelian Example: $\mathbf{D}_{3}$}

As an example of a non-abelian group we will work out the details for the group $G=\mathbf{D}_{3} \cong S_{3}$. It is generated by the elements $\tau$ and $\theta$ with defining relations 
$\theta^{3}=\tau^{2}=(\theta \tau)^{2}=1$. The conjugacy classes are $C_{1}=\{1\}, C_{\tau}=\left\{\tau, \tau \theta, \tau \theta^{2}\right\}$ and $C_{\theta}=\left\{\theta, \theta^{2}\right\}$. The stabilizer subgroups are given by $N_{1}=\mathbf{D}_{3}, N_{\tau}=\mathbf{Z}_{2}$ and $N_{\theta}=\mathbf{Z}_{3}$. There are three irreducible representations of $\mathbf{D}_{3}$ : two one-dimensional representations defined by $r(\tau)= \pm 1$ and $r(\theta)=1$, and a two-dimensional representation obtained by the embedding $D_{3} \subset O(2)$,

$$
r(\tau)=\left(\begin{array}{ll}
0 & 1 \\
1 & 0
\end{array}\right) \quad r(\theta)=\left(\begin{array}{ll}
0 & -1 \\
1 & -1
\end{array}\right) .
$$

This representation is easily verified to be irreducible. We will denote these representations as $1,1_{-}$and $\theta_{0}$ respectively. This notation will be explained in a moment. The stabilizer $N_{\theta}=\mathbf{Z}_{3}$ has three representations: $\theta_{i}(i=1,2,3)$, and $N_{\tau}=\mathbf{Z}_{2}$ has two: $\tau_{+}, \tau_{-}$.

So altogether we have 8 operators in the $\mathbf{D}_{3}$ orbifold. If we set $\sigma=1$, the matrix $S$ is given as in Table 1 .

Table 1. The matrix $S$, up to an overall factor $1 / 6$, for the $\mathbf{D}_{3}$ orbifold with $\sigma=1$. Here $\varepsilon_{\mu v}=4$ if $\mu=v$ and -2 otherwise $(\mu, v=0, \ldots, 3)$

\begin{tabular}{lrrlrr}
\hline & 1 & $1_{-}$ & $\theta_{v}$ & $\tau_{+}$ & $\tau_{-}$ \\
\hline 1 & 1 & 1 & 2 & 3 & 3 \\
$1_{-}$ & 1 & 1 & 2 & -3 & -3 \\
$\theta_{\mu}$ & 2 & 2 & $\varepsilon_{\mu v}$ & 0 & 0 \\
$\tau_{+}$ & 3 & -3 & 0 & 3 & -3 \\
$\tau_{-}$ & 3 & -3 & 0 & -3 & 3 \\
\hline
\end{tabular}

From this we read off the fusion rules

$$
\begin{gathered}
1_{-} \times 1_{-}=1, \quad \theta_{\mu} \times \theta_{\mu}=1+1_{-}+\theta_{\mu}, \quad \theta_{\mu} \times \theta_{v}=\sum_{\lambda \neq \mu, v} \theta_{\lambda}, \\
\tau_{+} \times \tau_{+}=\tau_{-} \times \tau_{-}=1+\sum_{\mu} \theta_{\mu}, \quad \tau_{+} \times \tau_{-}=1_{-}+\sum_{\mu} \theta_{\mu} .
\end{gathered}
$$

The remarkable symmetry in the $\theta_{\mu}$ can be understood, if we recall that for solvable groups, we can construct orbifolds by modding out by a sequence of abelian groups. If we first consider an orbifold obtained by modding out by the normal subgroup $\mathbf{Z}_{3} \triangleleft \mathbf{D}_{3}$, which possesses 9 operators, and then modding out by a $\mathbf{Z}_{2}\left(\mathbf{D}_{3} / \mathbf{Z}_{3} \cong \mathbf{Z}_{2}\right)$. The group $\mathbf{Z}_{2}$ acts by exchanging all the operators, except the identity, giving rise to the 4 operators $\theta_{\mu}$ (as will be discussed in the next section).

An explicit realization of a $\mathbf{D}_{3}$ orbifold with $\sigma=1$ can be constructed using the $E_{8} k=1$ model. The appropriate subgroup is $\mathbf{D}_{3} \subset S U(3)$, with $S U(3) \times E_{6} \subset E_{8}$. As an amusing exercise one can calculate the dimensions of the higher genus vector bundles, e.g. for a genus 2 surface one finds $\operatorname{dim} V_{2}=116$ - a result that can be checked by hand.

The general form of the interactions is found by solving the cohomology problem. We include the phases $\sigma(g \mid h)$ and solve the condition $\delta_{1} \delta_{2} \sigma=1$ modulo solutions of the form (4.17). In this case we find as the only independent variables $\sigma=\sigma(\theta \mid \theta)$ and $\beta=\sigma(\tau \mid \tau)$. Inserting this into $\delta_{1} \delta_{2} \sigma=1$ we obtain $\sigma^{9}=\beta^{2}=1$. 
This gives the $S$-matrix of Table 2,

Table 2. The general form of the matrix $S$ for the $\mathbf{D}_{3}$ orbifold

\begin{tabular}{rrrrrrrrr}
\hline & 1 & $1_{-}$ & $\theta_{0}$ & $\theta_{1}$ & $\theta_{2}$ & $\theta_{3}$ & $\tau_{+}$ & $\tau_{-}$ \\
\hline 1 & 1 & 1 & 2 & 2 & 2 & 2 & 3 & 3 \\
1. & 1 & 1 & 2 & 2 & 2 & 2 & -3 & -3 \\
$\theta_{0}$ & 2 & 2 & 4 & -2 & -2 & -2 & 0 & 0 \\
$\theta_{1}$ & 2 & 2 & -2 & $2 \alpha_{0}$ & $2 \alpha_{2}$ & $2 \alpha_{1}$ & 0 & 0 \\
$\theta_{2}$ & 2 & 2 & -2 & $2 \alpha_{1}$ & $2 \alpha_{0}$ & $2 \alpha_{2}$ & 0 & 0 \\
$\theta_{3}$ & 2 & 2 & -2 & $2 \alpha_{2}$ & $2 \alpha_{1}$ & $2 \alpha_{0}$ & 0 & 0 \\
$\tau_{+}$ & 3 & -3 & 0 & 0 & 0 & 0 & $3 \beta$ & $-3 \beta$ \\
$\tau_{-}$ & 3 & -3 & 0 & 0 & 0 & 0 & $-3 \beta$ & $3 \beta$ \\
\hline
\end{tabular}

with $\alpha_{i}=\sigma \omega^{i}+\overline{\sigma \omega}^{i}\left(\omega=e^{2 \pi i / 3}\right)$. We still have $S=S^{T}$ and $S^{2}=1$. The fusion rules are modified only by:

$$
\theta_{i} \times \theta_{i}=1+1_{-}+N_{i i}{ }^{k} \theta_{k}, \quad \theta_{i} \times \theta_{j}=\theta_{0}+N_{i j}{ }^{k} \theta_{k},
$$

where the integer coefficients $N_{i j}{ }^{k}$ are defined through $\alpha_{i} \alpha_{i}=2+N_{i i}{ }^{k} \alpha_{k}$ and $\alpha_{i} \alpha_{j}=-1+N_{i j}{ }^{k} \alpha_{k}$. This can be considered to be the general fusion algebra of an holomorphic $\mathbf{D}_{3}$ orbifold.

\section{Some Remarks on General Rational Orbifolds}

In this section we will consider rational conformal field theories with more than one chiral block. We suppose that the theory has a symmetry $G$, and we wish to mod out by some action of $G$. It turns out that the resulting operator algebra is far more difficult to analyze than the orbifolds built out of holomorphic theories, and we will make only some general remarks about them. Examples of such orbifolds which display the general features discussed in this section will be presented in the next section (in the context of $c=1$ models).

The fundamental difficulty in analyzing the orbifolds constructed from general RCFT's is that their modular properties do not seem to be completely dictated by the group structure and the operator algebra we started with. For example, we will have to consider partition functions of the form

$$
\operatorname{Tr}_{\left[\phi_{t}\right]} g q^{L_{0}}
$$

where $\left[\phi_{i}\right]$ is a chiral sector of the theory we started with. Under the modular transformation $S$, this gives a set of new characters. How do we organize these new characters? In the holomorphic case we had the group structure to guide us in the organization, but now with the operator algebra of the initial theory mixed in, one has to find an organizing principle for the characters and this does not seem clear.

What we will do is limit ourselves just to the counting of the operators in generic cases for the case of solvable groups. We will first do this for the case where $G=\mathbf{Z}_{k}$. Of course we can continue the modding out by a sequence of $\mathbf{Z}_{N}$ 's. Thus 
we will be able to do the counting for any solvable group. Non-abelian groups of this kind will be encountered in the next section in the context of $c=1$ models.

Let $g$ be the generator of $\mathbf{Z}_{k}$, where $g^{k}=1$. To begin with we will assume that $k$ is a prime. $g$ acts on the operator algebra by either permuting $k$ operators cyclically, or by acting trivially on some operators (this is so because we have chosen $k$ to be prime). Suppose the operator algebra we start with has $N$ chiral sectors. Let $n$ be the number of operators left fixed under the action of $g$, and $m$ be the number of groups of $k$ operators which are cyclically permuted by the action of $g$, then we have

$$
N=n+m k \text {. }
$$

We claim that the total number of operators $\tilde{N}$ that we will obtain by modding by $G$ is

$$
\tilde{N}=n k^{2}+m \text {. }
$$

To see this consider the untwisted sector first. This corresponds to considering linear combination of characters of the form

$$
\operatorname{Tr}_{\left[\phi_{0}\right]} g^{l} q^{L_{0}} .
$$

For the sectors which are fixed under the action of the modular group this results in $k$ different characters for each sector ${ }^{6}$. However, for the $m k$ sectors which are permuted by this action the above trace vanishes and we get nothing new. In fact, since the sectors which are mapped to each other must have the same character (as is required if the $g$ action which permutes them is a symmetry of the conformal theory), for each group of $k$ sectors which are permuted among each other we are left with only one character. In other words, under the action of $g$ the $k$ sectors are identified and should be counted as one. So from the untwisted sector we obtain $n k+m$ operators. Now consider the sectors twisted by $g$. These sectors are obtained by considering the modular transformation $S$ on the characters in (6.4). As we discussed there are $n$ independent non-vanishing characters in (6.4). So we obtain $n$ independent sectors each twisted by $g$. Each of these sectors has to be decomposed into representations of $\mathbf{Z}_{k}$ which are obtained by taking linear combination of the modular transformations $T^{l}$ acting on them for $l$ running from 1 to $k$. So in the first twisted sector we obtain $n k$ new chiral sectors. This story repeats in each sector and we finally obtain $(k-1) n k$ chiral sectors from the twisted sectors. Including the contribution of the untwisted sector we see that we have $\tilde{N}=n k^{2}+m$ operators, as was to be shown.

If $k$ is not a prime, the operators form orbits of size $l_{i}$ under the action of $g$, where $l_{i}$ divides $k$, and we have

$$
N=\sum_{i} l_{i}
$$

\footnotetext{
${ }^{6}$ Here we are assuming a generic case, where there is no reason for (6.4) to vanish for the sectors which are fixed by the action of $g$. There are examples of conformal theories where extra symmetries force this to be zero for some sectors
} 
By a simple modification of the argument presented above, it is easy to see that the total number of operators we obtain is

$$
\tilde{N}=\sum_{i}\left(k / l_{i}\right)^{2}
$$

This concludes our discussion for counting the number of operators in generic cases for solvable groups.

Even though we have not determined the operator algebra for the nonholomorphic case, there are some selection rules which are obvious. One is that group multiplication law dictates certain selection rules. Also the representation tensor products will indicate a selection rule (modulo the inclusion of tensor products with a one dimensional representation) as discussed for holomorphic theories. The ideas in this section will be illustrated in the light of $c=1$ conformal theories in the next section.

\section{Orbifolds at $c=1$}

In this section we will apply the results we derived in the previous sections in a study of orbifold CFT's with central charge $c=1$. These models have been thoroughly studied in the literature and many of their properties, for example the partition functions, have been explicitly calculated. However, these analyses did not explain the chiral structure, and hence it will be interesting to reconsider these models, and analyze them from a chiral point of view, i.e. give a description of their characters and fusion algebras. As such they will serve as an illustration, and, in particular for non-abelian groups, give indications for the general structure present in orbifold CFT's.

\section{7.a. $c=1$ Revisited}

In order to introduce some notations we will first briefly review some familiar facts of $c=1$ conformal field theory. More details can be found in [28-30].

Let us first consider the different symmetry algebras that occur at $c=1$. They will always contain the $c=1$ Virasoro algebra, which does not act freely but is known to possess null states at weights $h=\frac{1}{4} n^{2}$ with $n \in \mathbf{Z}$. Accordingly the Virasoro characters are given by the following expressions:

$$
\chi_{h}^{(\mathrm{Vir})}(q)= \begin{cases}\frac{1}{\eta(q)}\left[q^{\frac{1}{4} n^{2}}-q^{\frac{1}{4}(n+2)^{2}}\right], & \text { if } h=\frac{1}{4} n^{2}, n \in \mathbf{Z}, \\ \frac{1}{\eta(q)} q^{h}, & \text { otherwise. }\end{cases}
$$

It is a remarkable fact that no conformal field theory with $c \geqq 1$ (where we do not necessarily impose the condition of rationality) is known whose chiral algebra consists only of the Virasoro algebra, i.e. there always appear chiral scaling operators. This is in particular the case for $c=1$, so let us discuss the different chiral algebras that occur.

First we have of course the $U(1)$ current algebra, which is feely generated by a spin 1 field $j(z)$ and can be considered as the generic $c=1$ algebra. It is the chiral 
symmetry algebra of the Gaussian model, describing a free bosonic field $\phi$ possibly identified modulo $2 \pi R$, with $R$ the compactification radius. Its basic character equals $1 / \eta(q)$ since no null-states are present. The operator content of the $U(1)$ current algebra can now be determined by decomposing this expression into Virasoro characters using (7.1):

$$
\frac{1}{\eta(q)}=\sum_{n \in \mathbf{Z}_{\geqq 0}} \frac{1}{\eta(q)}\left[q^{n^{2}}-q^{(n+1)^{2}}\right]=\sum_{n \in \mathbf{Z}_{\geqq 0}} \chi_{n^{2}}^{(\mathrm{Vir})} .
$$

So the current algebra contains an infinite set of primary fields of $\operatorname{spin} n^{2}\left(n \in \mathbf{Z}_{\geq 0}\right)$. These chiral fields $j_{n^{2}}(z)$ can be expressed in (normal ordered) Schur polynomials in the current $j(z)$ and its (multiple) derivatives [31]. For example $j_{1}=j$ and the spin 4 field is given by

$$
j_{4}=j^{4}-2 j \partial^{2} j+\frac{3}{2}(\partial j)^{2} .
$$

The representations of the current algebra are the chiral vertex operators $\phi_{p}=e^{i p \varphi}$, with $j=i \partial \varphi$. The label $p$ corresponds to the global $U(1)$ charge. Since this is an additive quantity the fusion algebra reads simply

$$
\phi_{p} \times \phi_{p^{\prime}}=\phi_{p+p^{\prime}}
$$

and is isomorphic to $\mathbf{R}$.

The current algebra can be further enlarged if we include a vertex operator of integer spin $N$ [32]. (In fact, closure of the algebra tells one to include all vertex operators with momenta in $\sqrt{2 N} \mathbf{Z}$.) The algebra we obtain in this way will be denoted as $\mathscr{A}_{N}$. It is generated by the fields $j, V_{+}, V_{-}$, where $V_{ \pm}=e^{ \pm i \sqrt{2 N} \varphi}$. This extended symmetry occurs in the so-called rational Gaussian models, where $R^{2} \in \mathbf{Q}$. More precisely, if $\frac{1}{2} R^{2}=p / p^{\prime}$ with $\left(p, p^{\prime}\right)=1$ (no common divisor) then the maximal chiral algebra equals $\mathscr{A}_{N}$ with $N=p p^{\prime}$. Since the representations also have to be local with respect to $V_{ \pm}$, this chiral algebra possesses only a finite number of representations $\left[\phi_{k}\right]$, with primary fields $\phi_{k}=e^{i k \varphi / \sqrt{2 N}}$ with $k \in \mathbf{Z}_{2 N}$. Accordingly the fusion algebra reduces to $\mathbf{Z}_{2 N}$. Models described by $\mathscr{A}_{N}$ can perhaps be considered as the most trivial examples of rational CFT's. For later reference we give the characters and their modular properties under $S$ :

$$
\begin{gathered}
\chi_{k}(q)=\frac{1}{\eta(q)} \sum_{m \in Z} q^{(k+m 2 N)^{2} / 4 N}, \\
S: \chi_{k} \rightarrow \frac{1}{\sqrt{2 N}} \sum_{k^{\prime} \in \mathbf{Z}_{2 N}} e^{-i \pi k k^{\prime} / 2 N} \chi_{k^{\prime}} .
\end{gathered}
$$

A particular example of such an extended $U(1)$ algebra is $\mathscr{A}_{1}$, which equals the $S U(2)$ level 1 affine algebra. It possesses only two representations: the identity $[1]=\left[\phi_{0}\right]$ and the spinor $[\phi]=\left[\phi_{1}\right]$ with fusion algebra $\phi \times \phi=1$. The characters can be decomposed into Virasoro characters as

$$
\begin{aligned}
& \chi_{0}=\sum_{n \in \mathbf{Z}_{\geqq 0}}(2 n+1) \chi_{n^{2}}^{(\mathrm{Vir})}, \\
& \chi_{1}=\sum_{n \in \mathbf{Z}_{\geqq 0}}(2 n+2) \chi_{\left(n+\frac{1}{2}\right)^{2}}^{(\mathrm{Vir})},
\end{aligned}
$$


and we clearly see the $S U(2)$ multiplet structure appear with all integer and halfinteger representations occurring in respectively [1] and $[\phi]$.

In fact, this model gives a nice way to see the necessity of the existence of the primary fields $j_{n^{2}}$ in the $U(1)$ algebra. The vertex operator $e^{i n \sqrt{2} \varphi}$ is evidently a primary field with $S U(2)$ quantum numbers $j, m$ both equal to $n$ and conformal weight $h=n^{2}$. It is the highest weight of a $(2 n+1)$-dimensional multiplet, which contains in particular a primary field of charge $m=0$ and the same weight $h=n^{2}$. This field, being in the zero charge subsector, is necessarily a polynomial in the current and its derivatives and should be identified with the field $j_{n^{2}}$. Furthermore we can now easily calculate the OPE's

$$
j_{n_{1}^{2}}(z) j_{n_{2}^{2}}(0)=\sum_{n_{3}} c_{n_{1} n_{2} n_{3}} z^{n_{1}^{2}+n_{2}^{2}-n_{3}^{2}} j_{n_{3}^{2}}(0) .
$$

Since the $j_{n_{i}^{2}}$ form part of an $S U$ (2) multiplet, the OPE coefficients can be simply expressed in terms of the $S U$ (2) Clebsh-Gordan coefficients $C_{m_{1} m_{2} m_{3}}^{j_{1} j_{2} j_{3}}$ as

$$
c_{n_{1} n_{2} n_{3}}=C_{000}^{n_{1} n_{2} n_{3}} .
$$

Note that we have a constraint $n_{1}+n_{2}-n_{3}=0(\bmod 2)$ as a result of invariance under the parity transformation $j_{n^{2}} \rightarrow(-1)^{n} j_{n^{2}}$.

The only other chiral algebras known to exist at $c=1$ are orbifold algebras of the above. We essentially have two types of orbifolds. The generic Gaussian models only allow a $\mathbf{Z}_{2}$ orbifold, but in the case of the $S U$ (2) WZW model we can mod out by any discrete subgroup $G$ of $S O(3) \subset S U(2)$. The chiral algebras consist in both cases of the invariant subset of chiral fields. In order to discuss these algebras and their representations we will turn to the one-loop partition functions as calculated in [29].

We recall that all known $c=1$ models have partition functions that can be expressed as linear combinations of the Gaussian partition functions $\mathscr{Z}_{R}$,

$$
\mathscr{Z}_{R}=\frac{1}{\eta \bar{\eta}} \sum_{(p, \bar{p}) \in \Gamma R} q^{\frac{1}{2} p^{2}} \bar{q}^{\frac{1}{2} \bar{p}^{2}},
$$

with $R$ the compactification radius and

$$
\Gamma_{R}=R \mathbf{Z}\left(\begin{array}{l}
1 \\
1
\end{array}\right) \oplus \frac{1}{2 R} \mathbf{Z}\left(\begin{array}{c}
1 \\
-1
\end{array}\right)
$$

Of particular interest are the radii $R=r / N$ with $r$ the radius of the $k=1 S U(2)$ model (in our normalization $r=\sqrt{2}$ ). We will denote the corresponding partition functions as $\mathscr{Z}_{N}$. Using these expressions the partition functions of the orbifold models are written as follows. For the $\mathbf{Z}_{2}$ orbifold of a Gaussian model of radius $R$ we find

$$
\mathscr{Z}=\frac{1}{2} \mathscr{Z}_{R}+\mathscr{Z}_{2}-\frac{1}{2} \mathscr{Z}_{1} \text {. }
$$

As for the orbifolds of the $S U(2)$ WZW model, we recall that the discrete subgroups of $S O(3)$ are in one-to-one correspondence with the simply-laced Lie algebras. Accordingly we have two series: the groups $\mathbf{Z}_{N}$ and $\mathbf{D}_{N}$, and three exceptional non-abelian groups $T, O, I$, (the symmetry groups of the tetrahedron, 
octahedron and icosahedron). The corresponding partition functions have been calculated by Ginsparg [29]:

$$
\begin{array}{lll}
\mathbf{Z}_{N}: \mathscr{Z}=\mathscr{Z}_{N}, & j_{0}=1, \\
\mathbf{D}_{N}: \mathscr{Z}=\frac{1}{2} \mathscr{Z}_{N}+\mathscr{Z}_{2}-\frac{1}{2} \mathscr{Z}_{1}, & j_{0}=2, \\
T: \mathscr{Z}=\mathscr{Z}_{3}+\frac{1}{2} \mathscr{Z}_{2}-\frac{1}{2} \mathscr{Z}_{1}, & j_{0}=3, \\
O: \mathscr{Z}=\frac{1}{2} \mathscr{Z}_{4}+\frac{1}{2} \mathscr{Z}_{3}+\frac{1}{2} \mathscr{Z}_{2}-\frac{1}{2} \mathscr{Z}_{1}, & j_{0}=4, \\
I & : \mathscr{Z}=\frac{1}{2} \mathscr{Z}_{5}+\frac{1}{2} \mathscr{Z}_{3}+\frac{1}{2} \mathscr{Z}_{2}-\frac{1}{2} \mathscr{Z}_{1}, & j_{0}=6 .
\end{array}
$$

We will explain the numbers $j_{0}$ in a moment. Let us first briefly discuss the chiral algebras of these models. Since they are subalgebras of the $S U$ (2) current algebra, the algebras $\mathscr{A}_{1} / G$ should contain only fields of weight $h=j^{2}(j \in \mathbf{Z})$. The multiplicity of the spin $j^{2}$ field is equal to the number of times the identity representation of $G$ occurs in the branching of the isospin $j$ representation of $S O$ (3) into the irreducible representations of $G$. This can indeed be verified by explicit computation, using the fact that the multiplicity of chiral fields of $\operatorname{spin} j^{2}$ in the partition function $\mathscr{Z}_{N}$ is given by $1+2[j / N]$. Let $j_{0}$ be the smallest value of $j$ such that this multiplicity does not vanish, i.e. $j_{0}^{2}$ is the smallest conformal weight in the spectrum of the primary operator in the chiral algebra. Then the values of $j_{0}$ for the $S U(2)$ orbifolds are indicated in (7.14).

It is not difficult to show that the above list of partition functions exhaust all $c=1$ models whose partition function can be written as a linear sum of $\mathscr{Z}_{R}$ 's,

$$
\mathscr{Z}=\sum_{i} c_{i} \mathscr{Z}_{R_{i}},
$$

(see also [33].) Note that necessarily $\Sigma_{i} c_{i}=1$, since the identy occurs with multiplicity 1 . In order to prove this we have to distinguish two situations. First, we consider the case that the chiral algebra contains a spin 1 current $j(z)$. The condition that all multiplicities are non-negative leads now to two possibilities: we either have a Gaussian model with $\mathscr{Z}=\mathscr{Z}_{R}$ or we have a partition function of the form

$$
\mathscr{Z}=\frac{1}{2} \mathscr{Z}_{R_{1}}+\frac{1}{2} \mathscr{Z}_{R_{2}} \text {. }
$$

We can rule out the second possibility by the following argument. Since a $U(1)$ current subalgebra is present we can decompose (7.16) into the $U(1)$ characters $q^{\frac{1}{2} p^{2}} / \eta(q)$ with $p$ the global $U(1)$ charge. Here we used the fact that necessarily the stress-energy tensor is given by the Sugawara form $T(z)=\frac{1}{2} j(z)^{2}$. Now the partition function (7.16) has the property that the charges $p, \bar{p}$ are not summed over a lattice, since $\Gamma_{R_{1}}$ and $\Gamma_{R_{2}}$ are not compatible. This already points out a problem since $p$ is additively conserved. But furthermore it implies that $\mathscr{Z}$ contains characters with $p \neq 0$ and with multiplicity 1 . The corresponding operators should accordingly be self-conjugate which conflicts with the fact that conjugate fields carry the opposite charge $-p$. This proves the impossibility of (7.16).

So in order to find other than Gaussian models we have to exclude spin 1 fields. Since the multiplicity of the spin 1 field equals 3 for $\mathscr{Z}_{1}$ and 1 for all other $Z_{R}$, this implies that $\mathscr{Z}_{1}$ should always be included in our sum and that its coefficient should be $-\frac{1}{2}$. The coefficients in (7.15) are further restricted by applying the 
positivity condition for the chiral fields of dimension $j^{2}$ with $j>1$. It is an easy exercise to verify with the aid of Table 3 that the possible combinations are already restricted to the above cases just by requiring integer, non-negative multiplicities for $j \leqq 5$.

Table 3. The multiplicities of the weight $\left(j^{2}, 0\right)$ fields in the partition functions $\mathscr{Z}_{N}$

\begin{tabular}{llllllr}
\hline$j$ & 0 & 1 & 2 & 3 & 4 & 5 \\
\hline $\mathscr{Z}_{1}$ & 1 & 3 & 5 & 7 & 9 & 11 \\
$\mathscr{Z}_{2}$ & 1 & 1 & 3 & 3 & 5 & 5 \\
$\mathscr{Z}_{3}$ & 1 & 1 & 1 & 3 & 3 & 3 \\
$\mathscr{Z}_{4}$ & 1 & 1 & 1 & 1 & 3 & 3 \\
$\mathscr{Z}_{5}$ & 1 & 1 & 1 & 1 & 1 & 3 \\
$\mathscr{Z}_{R}$ & 1 & 1 & 1 & 1 & 1 & 1 \\
\hline
\end{tabular}

We further note that any orbifold partition function in (7.14) can be written as

$$
\mathscr{Z}=\frac{1}{2} Z_{n_{1}}+\frac{1}{2} \mathscr{Z}_{n_{2}}+\frac{1}{2} \mathscr{Z}_{n_{3}}-\frac{1}{2} \mathscr{Z}_{1},
$$

where $n_{1}, n_{2}, n_{3}$ are three positive integers satisfying

$$
\frac{1}{n_{1}}+\frac{1}{n_{2}}+\frac{1}{n_{3}}=1+\frac{2}{N}>1
$$

This last condition is exactly the equation that classifies all discrete $S O$ (3) subgroups, with $N$ the order of the group, or the simply-laced Lie algebras, with the $n_{i}$ the number of nodes on each branch of the Dynkin diagram. The above inequality can be understood in the context of $c=1$ conformal field theory by calculating the multiplicity of the spin $j$ field in (7.17) in the limit $j \rightarrow \infty$, and requiring it to be positive.

We have seen that the above expressions for the partition functions completely obscure the analytical structure. We cannot simply read off the characters, i.e. the partition functions are not written in the canonical form $\mathscr{Z}=\Sigma \chi_{i} \bar{\chi}_{\bar{i}}$. In order to derive the characters we will exploit the fact that the partition functions $\mathscr{Z}_{N}$ can be written as

$$
\mathscr{Z}_{N}(q, \bar{q})=\sum_{\substack{\alpha \in \frac{1}{2 V} \mathbf{Z}_{2,} \\
\beta \in \frac{1}{N}}} \frac{1}{N}\left|\vartheta\left[\begin{array}{l}
\alpha \\
\beta
\end{array}\right](q)\right|^{2},
$$

where we introduced a generalized theta function

$$
\vartheta\left[\begin{array}{l}
\alpha \\
\beta
\end{array}\right](q)=\frac{1}{\eta(q)} \vartheta\left[\begin{array}{l}
\alpha \\
\beta
\end{array}\right](0 \mid 2 \tau)=\frac{1}{\eta(q)} \sum_{m \in \mathbf{Z}} q^{(m+\alpha)_{2}} e^{2 \pi i m \beta} .
$$

Note that the ף's have an interpretation as twisted sectors of the $S U(2)$ model - an interpretation we will discuss in detail in Sect.7.c. These $\vartheta$-functions enjoy the following modular transformation properties:

$$
\begin{aligned}
& S: \vartheta\left[\begin{array}{l}
\alpha \\
\beta
\end{array}\right] \rightarrow \frac{1}{\sqrt{2}}\left\{e^{-2 \pi i \alpha \beta} \vartheta\left[\begin{array}{c}
\beta / 2 \\
-2 \alpha
\end{array}\right]+e^{-2 \pi i \alpha(\beta+1)} \vartheta\left[\begin{array}{c}
(\beta+1) / 2 \\
-2 \alpha
\end{array}\right]\right\}, \\
& T: \vartheta\left[\begin{array}{l}
\alpha \\
\beta
\end{array}\right] \rightarrow e^{2 \pi i\left(\alpha^{2}-\frac{c}{24}\right)} \vartheta\left[\begin{array}{c}
\alpha \\
\beta+2 \alpha
\end{array}\right] .
\end{aligned}
$$


We can now in principle try to decompose the partition function into holomorphic blocks that are linear sums of the $\vartheta$ 's. These will be conjectured to be the generalized characters of the extended chiral algebra relevant for the particular model. The behavior under modular transformations will give us the fusion rules. We will give the explicit calculations in next sections.

\section{7.b. Rational $\mathbf{Z}_{2}$ Orbifolds}

We start our analysis by considering the $\mathbf{Z}_{2}$ orbifold model, i.e. a scalar field $\varphi$ defined $\bmod 2 \pi R$ and identified by $l: \varphi \rightarrow-\varphi$. The partition function is given by (7.13). We will choose $R^{2}$ to be rational, so that our original algebra equals $\mathscr{A}_{N}$. The operator content of the algebra $\mathscr{A}_{N} / \mathbf{Z}_{2}$ is easy to describe. It consists simply of all the elements of $\mathscr{A}_{N}$ invariant under the involution $l$. If we use the fact that $l: j_{n^{2}} \rightarrow(-1)^{n} j_{n^{2}}$, then it is evident that $\mathscr{A} / \mathbf{Z}_{2}$ contains in particular all fields $j_{n^{2}}$ with $n$ even. This subalgebra is generated by $j_{4}$, as can be seen from (7.9). It is not difficult to verify that the total chiral algebra is generated by the fields

$$
T, j_{4}, \cos \sqrt{2 N} \varphi
$$

of respectively spin 2,4 , and $N$.

In order to determine the representations of this algebra we try to decompose the partition function into holomorphic and anti-holomorphic blocks. After substituting (7.19) this results in $N+7$ operators. Their respective characters and conformal weights are given below (here $\chi_{k}$ denote the $\mathscr{A}_{N}$ characters (7.5))

$$
\begin{aligned}
1: \chi & =\frac{1}{2} \chi_{0}+\frac{1}{2} \vartheta\left[\begin{array}{c}
0 \\
1 / 2
\end{array}\right], & h & =0, \\
j & : \chi=\frac{1}{2} \chi_{0}-\frac{1}{2} \vartheta\left[\begin{array}{c}
0 \\
1 / 2
\end{array}\right], & h & =1, \\
\phi_{N}^{i}: \chi & =\frac{1}{2} \chi_{N}, \quad(i=1,2), & h & =\frac{1}{4} N, \\
\phi_{k}: \chi & =\chi_{k}, \quad(k=1, \ldots, N-1), & h & =k^{2} / 4 N, \\
\sigma_{i}: \chi & : \chi \frac{1}{2} \vartheta\left[\begin{array}{c}
1 / 4 \\
0
\end{array}\right]+\frac{1}{2} \vartheta\left[\begin{array}{c}
1 / 4 \\
1 / 2
\end{array}\right] \quad(i=1,2), & h & =\frac{1}{16}, \\
\tau_{i}: \chi & : \chi \frac{1}{2} \vartheta\left[\begin{array}{c}
1 / 4 \\
0
\end{array}\right]-\frac{1}{2} \vartheta\left[\begin{array}{l}
1 / 4 \\
1 / 2
\end{array}\right] \quad(i=1,2), & h & =\frac{9}{16} .
\end{aligned}
$$

We can give the following interpretation of this operator content. Our original model consisted of the representations $\left[\phi_{k}\right]\left(k \in \mathbf{Z}_{2 N}\right)$. The $\mathbf{Z}_{2}$ transformation $l$ acts on these representations as

$$
l:\left[\phi_{k}\right] \rightarrow\left[\phi_{2 N-k}\right] .
$$

So it acts as an inner automorphism for the representations $\left[\phi_{0}\right]$ and $\left[\phi_{N}\right]$. These are the analogues of the fixed points that occur in the different orbifold models featured in string compactification models. According to Sect. 6 we consequently expect the following operators. The identity will split in an invariant part [1] and a non-invariant part $[j]$, whose primary field is the current $j=i \partial \varphi$. The same will happen for $\left[\phi_{N}\right]$ and we will denote these two representations as $\left[\phi_{N}^{i}\right]$. Furthermore, we will have 2 twisted sectors, each giving rise to 2 operators, corresponding to the trivial and the non-trivial representation of $\mathbf{Z}_{2}$. This accounts for the fields $\left[\sigma_{i}\right]$ and $\left[\tau_{i}\right]$. The remaining representations $\left[\phi_{k}\right]$, on which $l$ acts as an outer automorphism, are pairwise combined into an invariant operator, which corresponds to the vertex operator $\cos \frac{k}{\sqrt{2 N}} \varphi$. 
Now that we have identified the spectrum, we want to discuss the possible interactions. With the formulas given in the previous section one can easily derive the action of $S$ on the above characters. In this reconstruction of the modular transformation one should bear in mind the constraints of symmetry, unitarity and a closed operator product. It turns out that in order to satisfy all these demands we must distinguish between the cases $N$ odd and $N$ even. In the latter case the two representations $\left[\phi_{N}^{1}\right]$ and $\left[\phi_{N}^{2}\right]$ are self-conjugate, while in the former case they are each other's conjugate. For the case $N$ even the matrix $S$ is given in Table 4.

Table 4. The matrix $S$ (up to an overall factor $\frac{1}{\sqrt{8 N}}$ ) for the $\mathbf{Z}_{2}$ orbifold of the $\mathbf{Z}_{2 N}$ Gaussian model in the case $N$ even. (Here $\sigma_{i j}=2 \delta_{i j}-1$ )

\begin{tabular}{llllllc}
\hline & 1 & $j$ & $\phi_{N}^{j}$ & $\phi_{k^{\prime}}$ & $\sigma_{J}$ & $\tau_{j}$ \\
\hline 1 & 1 & 1 & 1 & 2 & $\sqrt{N}$ & $\sqrt{N}$ \\
$j$ & 1 & 1 & 1 & 2 & $-\sqrt{N}$ & $-\sqrt{N}$ \\
$\phi_{N}^{i}$ & 1 & 1 & 1 & $2(-1)^{k^{\prime}}$ & $\sigma_{i j} \sqrt{N}$ & $\sigma_{i j} \sqrt{N}$ \\
$\phi_{k}$ & 2 & 2 & $2(-1)^{k}$ & $4 \cos \pi \frac{k k^{\prime}}{2 N}$ & 0 & 0 \\
$\sigma_{i}$ & $\sqrt{N}$ & $-\sqrt{N}$ & $\sigma_{i j} \sqrt{N}$ & 0 & $\delta_{i j} \sqrt{2 N}$ & $-\delta_{i j} \sqrt{2 N}$ \\
$\tau_{i}$ & $\sqrt{N}$ & $-\sqrt{N}$ & $\sigma_{\imath \jmath} \sqrt{N}$ & 0 & $-\delta_{i j} \sqrt{2 N}$ & $\delta_{i j} \sqrt{2 N}$ \\
\hline
\end{tabular}

The corresponding fusion algebra can be directly deduced. We give only the relevant relations. Other relations follow from associativity. First the elements $1, j$, $\phi_{N}^{i}$ generate a $\mathbf{Z}_{2} \times \mathbf{Z}_{2}$ subalgebra

$$
\begin{aligned}
j \times j & =1, \\
\phi_{N}^{i} \times \phi_{N}^{i} & =1, \\
\phi_{N}^{1} \times \phi_{N}^{2} & =j .
\end{aligned}
$$

The vertex operators $\phi_{k}$ have a fusion algebra consistent with their interpretation as $\cos \frac{k}{\sqrt{2 N}} \varphi$,

$$
\begin{aligned}
\phi_{k} \times \phi_{k^{\prime}} & =\phi_{k+k^{\prime}}+\phi_{k-k^{\prime}} \quad\left(k^{\prime} \neq k, N-k\right), \\
\phi_{k} & \times \phi_{k}=1+j+\phi_{2 k}, \\
\phi_{N-k} & \times \phi_{k}=\phi_{2 k}+\phi_{N}^{1}+\phi_{N}^{2}, \\
j & \times \phi_{k} \\
& =\phi_{k} .
\end{aligned}
$$

The twist fields generate all vertex operators through the relations

$$
\begin{aligned}
\sigma_{i} \times \sigma_{i} & =1+\phi_{N}^{i}+\sum_{k \text { even }} \phi_{k}, \\
\sigma_{1} \times \sigma_{2} & =\sum_{k \text { odd }} \phi_{k} .
\end{aligned}
$$

The operator product structure of the $\tau_{i}$ can be easily deduced using

$$
j \times \sigma_{i}=\tau_{i} .
$$


In the case $N$ odd we find the matrix elements of $S$ as given in Table 5 .

Table 5. The matrix $S$ as in Table 4 in the case $N$ odd

\begin{tabular}{lllllll}
\hline & 1 & $j$ & $\phi_{N}^{j}$ & $\phi_{k^{\prime}}$ & $\sigma_{j}$ & $\tau_{j}$ \\
\hline 1 & 1 & 1 & 1 & 2 & $\sqrt{N}$ & $\sqrt{N}$ \\
$j$ & 1 & 1 & 1 & 2 & $-\sqrt{N}$ & $-\sqrt{N}$ \\
$\phi_{N}^{i}$ & 1 & 1 & -1 & $2(-1)^{k^{\prime}}$ & $i \sigma_{i j} \sqrt{N}$ & $i \sigma_{i j} \sqrt{N}$ \\
$\phi_{k}$ & 2 & 2 & $2(-1)^{k}$ & $4 \cos 2 \pi \frac{k k^{\prime}}{2 N}$ & 0 & 0 \\
$\sigma_{i}$ & $\sqrt{N}$ & $-\sqrt{N}$ & $i \sigma_{i j} \sqrt{N}$ & 0 & $e^{\pi i \sigma_{i j} / 4} \sqrt{N}$ & $-e^{\pi i \sigma_{i j} / 4} \sqrt{N}$ \\
$\tau_{\imath}$ & $\sqrt{N}$ & $-\sqrt{N}$ & $i \sigma_{i j} \sqrt{N}$ & 0 & $-e^{\pi i \sigma_{i j} / 4} \sqrt{N}$ & $e^{\pi i \sigma_{i j} / 4} \sqrt{N}$ \\
\hline
\end{tabular}

As mentioned before, this results in a somewhat different fusion algebra. The operator algebra of $1, j$ and $\phi_{N}^{i}$ now equals $\mathbf{Z}_{4}$

$$
\begin{aligned}
j \times j & =1, \\
\phi_{N}^{1} \times \phi_{N}^{2} & =1, \\
\phi_{N}^{i} \times \phi_{N}^{i} & =j,
\end{aligned}
$$

and for the twist fields one obtains

$$
\begin{aligned}
\sigma_{i} \times \sigma_{i} & =\phi_{N}^{i}+\sum_{k \text { odd }} \phi_{k}, \\
\sigma_{1} \times \sigma_{2} & =1+\sum_{k \text { even }} \phi_{k} .
\end{aligned}
$$

The fusion algebra of the vertex operators $\phi_{k}$ is left unchanged.

There are some special $\mathbf{Z}_{2}$ orbifold models, where we can check these fusion rules. First for $N=1$ the algebra contains one spin 1 current, and the model should reduce to a Gaussian model, more precisely the $\mathbf{Z}_{8}$ rational Gaussian model [28]. One can verify that this equivalence is indeed found, using the above fusion rules. In particular we find that in this case the twist fields $\sigma_{i}$ are represented as vertex operators, and accordingly should be mapped to each other by charge conjugation.

For $N=2$, where an extra spin 2 field is present besides the stress-energy tensor, the $\mathbf{Z}_{2}$ orbifold equals two decoupled Ising models. So we expect $3 \times 3$ operators, which corresponds to our counting $N+7$. Also the fusion rules agree with those of the Ising model. We can conclude that the algebra $\mathscr{A}_{2} / \mathbf{Z}_{2}$ is isomorphic to the tensor product of two $c=\frac{1}{2}$ Virasoro algebra. Note that in this cases the spin fields - given by the two Ising spins - are indeed self-conjugate.

The next case, $N=3$, corresponds to the $\mathbf{Z}_{4}$ parafermion theory. These parafermionic models can be regarded as the simplest minimal models of a class of chiral algebras, which are obtained by adjoining to the Virasoro algebra higher spin primary fields that correspond to Casimir operators of a simply laced Lie group [34]. In particular, for the $\mathbf{Z}_{n}$ parafermionic model the Lie group equals $S U(n)$. So in the case $n=4$ we expect a spin 3 and 4 field to generate the algebra 
$\mathscr{A}_{3} / \mathbf{Z}_{2}$. These fields are easily identified as $j_{4}$ and $\cos \sqrt{6} \varphi$. The Kac formula for the $S U$ (4) coset models gives the weights $\frac{1}{16}, \frac{1}{12}, \frac{1}{3}, \frac{9}{16}, \frac{3}{4}, 1$, in exact agreement with the spectrum given above.

We postpone a discussion of the case $N=4$, that corresponds to the 4 state Potts model, to the next section. We should further mention that $N=6$ gives us the discrete superconformal model at $c=1$. In fact the algebra $\mathscr{A}_{6} / \mathbf{Z}_{2}$ gives us the bosonic projection of the $N=1$ superconformal algebra. This algebra is consequently generated by a spin 4 and 6 field.

It is also interesting to consider the case $N=\infty$. In that case we obtain a symmetry algebra present in all the $\mathbf{Z}_{2}$ orbifold models, independent of the radius of compactification, and equals the maximal algebra for the case when $R^{2}$ is irrational. It consists of the fields $j_{n^{2}}$ with $n$ even, and is generated by the field $j_{4}$. Its representations are

$$
1, \quad j=i \partial \varphi, \quad \cos p \varphi(p>0), \quad \sigma_{i}, \quad \tau_{i} .
$$

However, the fusion algebra is not well-defined, since we cannot determine the conjugation properties of the twist fields. A possible solution to this problem would be to keep only one pair $(\sigma, \tau)$ as irreducible representations, which then should be included with multiplicity 2 . Furthermore, this is a case of a model that is not even quasi-rational [11], since the fusion of two twist fields will produce all vertex operators

$$
\sigma \times \sigma=1+\sum_{p>0} \cos p \varphi .
$$

This concludes our analysis of the $\mathbf{Z}_{2}$ case. We will turn now to the more complicated groups.

\section{7.c. The $S U(2)$ Orbifolds}

We will now discuss the orbifold models of the $S U(2)_{k=1}$ WZW model. Since the orbifolds based on the cyclic and dihedral subgroups $\mathbf{Z}_{N}, \mathbf{D}_{N}$ are equivalent to particular rational Gaussian respectively $\mathbf{Z}_{2}$ orbifold models, this will effectively only produce new RCFT's in the case of the three polyhedral subgroups $T, O, I$. We will however also discuss the $\mathbf{D}_{N}$ models, since from this point of view they give examples of non-abelian orbifolds.

The $S U(2)$ model has two blocks [1] and $[\phi]$, with $\mathbf{Z}_{2}$ operator algebra $\phi \times \phi=1$. Since the sector $[\phi]$ contains half-integer spin $S U(2)$ representations, the action of $G$ within $[\phi]$ will be projective. This causes no problems. In the general case where $G$ acts as an inner automorphism in more than one representation $\left[\phi_{i}\right]$, the chiral action of $G$ can be projective in all representations except for the identity sector, where the vacuum state should be left invariant. Furthermore, the cocycles that occur should respect the fusion algebra, i.e. if we have a cocycle $c_{i}(g, h)$ in the action on the representation $\left[\phi_{i}\right]$, then

$$
c_{i}(g, h) c_{j}(g, h)=c_{k}(g, h) \quad \text { if } N_{i j k} \neq 0 .
$$

So indeed for the algebra $\phi^{2}=1$ a cocycle $c(g, h)= \pm 1$ is allowed.

In this respect it is convenient to consider the lift $\hat{G} \subset S U(2)$, defined by

$$
1 \rightarrow \mathbf{Z}_{2} \rightarrow \hat{G} \stackrel{\pi}{\rightarrow} G \rightarrow 1,
$$


where $\pi$ is the projection $S U(2) \rightarrow S O(3)$. Elements of $\hat{G}$ will be denoted as $\hat{g}$, with $\pi(\hat{g})=g \in G$ and $\pi^{-1}(g)=\{\hat{g}, c \hat{g}\}$. Here $c$ denotes the generator of the center $\mathbf{Z}_{2}$ of $S U(2)$.

It is a matter of taste whether one considers the $S U(2)$ orbifolds as $G$ or $\hat{G}$ orbifolds. If we adopt the latter point of view, we have the extra condition that the generator of the center $c \in \hat{G}$ acts as +1 and -1 in the representations [1] and $[\phi]$ respectively.

Let us now discuss the operator content of these $S U(2) / G$ orbifolds. We will first treat the untwisted sector. Both the representations [1] and [ $\phi]$ are evidently fixed points under the action of any subgroup $G$. Accordingly they will split up in smaller blocks that are labelled by the representations. The description is most conveniently done in terms of the representations $r_{\alpha}$ of $\hat{G}$. The identity sector [1] will give rise to blocks indexed by the irreducible representations $r_{\alpha}^{+}$of $\hat{G}$ that satisfy $r_{\alpha}^{+}(c)=1$, i.e. the (linear) representations of $G$. Similarly the blocks produced by spinor $[\phi]$ correspond to representations that obey $r_{\alpha}^{-}(c)=-1$, and are accordingly genuine projective representations of $G$. Note that consequently there is a one-to-one correspondence between the untwisted operators in the $S U(2) / G$ model and the nodes in the corresponding extended Dynkin diagram. Furthermore the fusion algebra of these untwisted operators just equals the representation ring of $\hat{G}$.

The characters of these operators are also easily written down. The operators coming from the identity sector [1], giving rise to the characters

$$
\chi_{\alpha}^{+}=\frac{1}{|G|} \sum_{h \in G} \varrho_{\alpha}^{+}(h) h \square_{1} .
$$

This is completely analogous to the chiral case. The characters of the operators that descend from the $[\phi]$ sector read in an evident notation

$$
\chi_{\alpha}^{-}=\frac{1}{|G|} \sum_{h \in G} \varrho_{\alpha}^{-}(h) h \square_{\phi} .
$$

We can actually calculate these blocks by bosonization [29]. The action of any element $h \in G$ can be represented as a shift $\beta \in \frac{1}{N} \mathbf{Z}_{N}$. This gives us the following expressions for the chiral blocks:

$$
h \square_{1}=\vartheta\left[\begin{array}{l}
0 \\
\beta
\end{array}\right], \quad h \square_{\phi}=\vartheta\left[\begin{array}{c}
1 / 2 \\
\beta
\end{array}\right] .
$$

We now have to include the twist fields. As we stated in the previous section a general prescription for the counting of twist fields and the determination of the correct fusion rules in an orbifold model based on an arbitrary RCFT is still lacking. However in this case we have explicit partition functions available, and we can actually calculate the characters. The results of this calculation, the details of which we will give in the next section for some examples, can be neatly summarized as follows. The number of twist fields is given by

$$
N_{\mathrm{twist}}=\sum_{i} n_{i}\left(n_{i}-1\right)
$$


where the $n_{i}$ are the integers of (7.18). Note that $n(n-1)$ is exactly the number of twisted states in a $\mathbf{Z}_{n}$ orbifold. Thus we obtain the following number of representations in the $S U(2) / G$ orbifolds:

$$
\mathbf{Z}_{N}: 2 N^{2}, \quad \mathbf{D}_{N}: N^{2}+7, \quad T: 21, \quad O: 28, \quad I: 37 .
$$

In the case of solvable groups we can also determine the correct number of twist operators using the explicit action of the different abelian group that is featured in the decomposition series. The only non-solvable group in this case is the icosahedral group $I \cong A_{5}$. The total number of operators we obtain in this way using the composition series

$$
\mathbf{Z}_{N} \triangleleft \mathbf{D}_{N}, \quad \mathbf{Z}_{2} \triangleleft \mathbf{D}_{2} \triangleleft T \triangleleft O,
$$

is consistent with above counting, as we will see in the next section.

We can give the following heuristic argument for the counting of the twist fields and the construction of their characters. The $S U(2)_{k=1}$ model can be viewed as a projection of a single block, non-local theory, where the operator $\phi$ is adjoined to the chiral algebra. The projection is onto elements even under the action of the center $c$. This creates the separate representations [1] and [ $\phi]$. We now construct the orbifold model, by first modding out the single block model by the group $\hat{G}$ and projecting on even states afterwards. According to this argument the twist sectors are labelled by the classes of $\hat{G}$. However, not all representations of the stabilizer occur, since we again have to project on those sectors that are even under the $\mathbf{Z}_{2}$ grading. This gives as the total number of operators, half the number in the holomorphic $\hat{G}$ orbifold. Note that in this picture the sector twisted by $c$ corresponds to $[\phi]$. With $N_{\hat{g}}$ the projection of the stabilizer of $\hat{g}$ into $S O(3)$, the expressions for the corresponding characters now read

$$
\chi_{\alpha}^{\hat{g}}=\frac{1}{\left|N_{\hat{g}}\right|} \sum_{h \in N_{g}} \varrho_{\alpha}^{\hat{g}}(h) h \underset{\widehat{g}}{\square} .
$$

We can again calculate these chiral blocks by bosonization, since we can always represent two commuting elements $\hat{g}$ and $h$ as shifts $\alpha \in \frac{1}{2 N} \mathbf{Z}_{2 N}, \beta \in \frac{1}{N} \mathbf{Z}_{N}$. Accordingly we can represent the block as

$$
h \square_{\hat{g}}=\vartheta\left[\begin{array}{l}
\alpha \\
\beta
\end{array}\right] .
$$

This representation also account for some features of the fusion algebra of the twisted operators. We will see an overall selection rule due to the class algebra of $\hat{G}$. Furthermore, the representations will decompose similarly as in the holomorphic case, if we take into account the phases $\sigma$ that can be read off from the modular properties of the above characters.

\section{7.d. Examples}

In this section we present the results of some more detailed calculations for specific examples, that confirm the prescription we gave in the previous section. 
As a first example let us consider the group $G=\mathbf{D}_{2}$. The model $S U(2) / \mathbf{D}_{2}$ is known to correspond to the 4-state Potts model at criticality. The group $\mathbf{D}_{2}$ acts on the $S U(2)$ currents $j_{a}(z)$ by

$$
r_{b}: j_{a} \rightarrow \varepsilon_{a b} j_{a}
$$

where the symbol $\varepsilon_{a b}$ is defined to be 1 if $a=b$, and -1 otherwise. The 4-state Potts model corresponds to the rational $\mathbf{Z}_{2}$ orbifold model with $N=4$. So we have already the prescription of Sect. 7.b to calculate the characters and fusion rules. We will rewrite the results in such a way that they can be seen to confirm the general prescriptions we gave for $S U(2)$ orbifolds. The partition functions can be decomposed in the following characters

$$
\begin{aligned}
1: \chi & =\frac{1}{4} \vartheta\left[\begin{array}{c}
0 \\
0
\end{array}\right]+\frac{3}{4} \vartheta\left[\begin{array}{c}
0 \\
1 / 2
\end{array}\right], & h & =0, \\
j_{a}: \chi & =\frac{1}{4} \vartheta\left[\begin{array}{c}
0 \\
0
\end{array}\right]-\frac{1}{4} \vartheta\left[\begin{array}{c}
0 \\
1 / 2
\end{array}\right], & h & =1, \\
\phi: \chi & =\frac{1}{2} \vartheta\left[\begin{array}{c}
1 / 2 \\
0
\end{array}\right], & h & =\frac{1}{4}, \\
\sigma_{a}: \chi & =\frac{1}{2} \vartheta\left[\begin{array}{c}
1 / 4 \\
0
\end{array}\right]+\frac{1}{2} \vartheta\left[\begin{array}{c}
1 / 4 \\
1 / 2
\end{array}\right], & h & =\frac{1}{16}, \\
\tau_{a}: \chi & =\frac{1}{2} \vartheta\left[\begin{array}{c}
1 / 4 \\
0
\end{array}\right]-\frac{1}{2} \vartheta\left[\begin{array}{c}
1 / 4 \\
1 / 2
\end{array}\right], & h & =\frac{9}{16} .
\end{aligned}
$$

From the modular properties of the blocks $\vartheta\left[\begin{array}{l}\alpha \\ \beta\end{array}\right]$ we can calculate the behavior of the characters under the modular transformation $S$. Up to an overall factor $\frac{1}{\sqrt{32}}$ we find the matrix $S$ as in Table 6 .

Table 6. The matrix $S_{i j}$ for $S U(2) / \mathbf{D}_{2}$ orbifold

\begin{tabular}{llllll}
\hline & 1 & $j_{b}$ & $\phi$ & $\sigma_{b}$ & \multicolumn{1}{c}{$\tau_{b}$} \\
\hline 1 & 1 & 1 & 2 & 2 & 2 \\
$j_{a}$ & 1 & 1 & 2 & $2 \varepsilon_{a b}$ & $2 \varepsilon_{a b}$ \\
$\phi$ & 2 & 2 & -4 & 0 & 0 \\
$\sigma_{a}$ & 2 & $2 \varepsilon_{a b}$ & 0 & $\delta_{a b} \sqrt{8}$ & $-\delta_{a b} \sqrt{8}$ \\
$\tau_{a}$ & 2 & $2 \varepsilon_{a b}$ & 0 & $-\delta_{a b} \sqrt{8}$ & $\delta_{a b} \sqrt{8}$ \\
\hline
\end{tabular}

By applying (7.21) we can read off from Table 6 what the fusion algebra of the 4 state Potts model is. We find

$$
\begin{aligned}
j_{a} \times j_{a} & =1, \\
j_{a} \times j_{b} & =j_{c}, \quad a \neq b \neq c, \\
\phi \times \phi & =1+\sum_{c} j_{c}, \\
\sigma_{a} \times \sigma_{a} & =1+\phi+j_{a}, \\
\sigma_{a} \times \sigma_{b} & =\sigma_{c}+\tau_{c}, \\
\sigma_{a} \times \tau_{a} & =\phi+\sum_{c \neq a} j_{c} .
\end{aligned}
$$

In order to see that the above structure indeed confirms our general argument, we consider the representation and class algebra of the lift of $\mathbf{D}_{2}$ into $S U(2): \hat{\mathbf{D}}_{2} \cong Q$, 
the quaternionic group. If we denote the 5 irreducible representations of $Q$ as $1, j_{a}$, $\phi$, then the representation algebra of $Q$ is indeed equal to the corresponding subalgebra of the above fusion rules. Furthermore $Q$ has 5 conjugacy classes $1, \phi$, $\sigma_{a}$. The projections of the stabilizers of the elements $\sigma_{a}$ into $\mathbf{D}_{2}$ are isomorphic to $\mathbf{Z}_{2}$. This accounts for the pairs of twist fields $\sigma_{a}, \tau_{a}$. The class algebra is given by

$$
\varphi^{2}=1, \quad \sigma_{1} \sigma_{2}=\sigma_{3}, \quad \sigma_{a}^{2}=1+\phi .
$$

This algebra can indeed be recognized as an overall selection rule in the fusion algebra.

As a less trivial example consider the tetrahedron group $T \cong A_{4}$. By explicit computation we found the following characters:

$$
\begin{array}{lll}
1_{0} & : \chi=\frac{1}{12} \vartheta\left[\begin{array}{l}
0 \\
0
\end{array}\right]+\frac{1}{4} \vartheta\left[\begin{array}{c}
0 \\
1 / 2
\end{array}\right]+\frac{2}{3} \vartheta\left[\begin{array}{c}
0 \\
1 / 3
\end{array}\right], & h=0, \\
j & : \chi=\frac{1}{4} \vartheta\left[\begin{array}{c}
0 \\
0
\end{array}\right]-\frac{1}{4} \vartheta\left[\begin{array}{c}
0 \\
1 / 2
\end{array}\right], & h=1, \\
1_{1}, 1_{2}: & : \chi=\frac{1}{12} \vartheta\left[\begin{array}{c}
0 \\
0
\end{array}\right]+\frac{1}{4} \vartheta\left[\begin{array}{c}
0 \\
1 / 2
\end{array}\right]-\frac{1}{3} \vartheta\left[\begin{array}{c}
0 \\
1 / 3
\end{array}\right], & h=4, \\
\phi_{0} & : \chi=\frac{1}{6} \vartheta\left[\begin{array}{c}
1 / 2 \\
0
\end{array}\right]-\frac{2}{3} \omega \vartheta\left[\begin{array}{l}
1 / 2 \\
1 / 3
\end{array}\right], & h=\frac{1}{4}, \\
\phi_{1}, \phi_{2}: \chi=\frac{1}{6} \vartheta\left[\begin{array}{c}
1 / 2 \\
0
\end{array}\right]+\frac{1}{3} \omega \vartheta\left[\begin{array}{c}
1 / 2 \\
1 / 3
\end{array}\right], & h=\frac{9}{4}, \\
\sigma & : \chi=\frac{1}{2} \vartheta\left[\begin{array}{c}
1 / 4 \\
0
\end{array}\right]+\frac{1}{2} \vartheta\left[\begin{array}{c}
1 / 4 \\
1 / 2
\end{array}\right], & h=\frac{1}{16}, \\
\tau & : \chi=\frac{1}{2} \vartheta\left[\begin{array}{c}
1 / 4 \\
0
\end{array}\right]-\frac{1}{2} \vartheta\left[\begin{array}{l}
1 / 4 \\
1 / 2
\end{array}\right], & h=\frac{9}{16}, \\
\omega_{i}^{ \pm} & : \chi=\frac{1}{3} \vartheta\left[\begin{array}{c}
1 / 3 \\
0
\end{array}\right]+\omega^{i} \vartheta\left[\begin{array}{l}
1 / 3 \\
1 / 3
\end{array}\right]+\varpi^{i} \vartheta\left[\begin{array}{l}
1 / 3 \\
2 / 3
\end{array}\right], & h=\frac{1}{9}, \frac{4}{9}, \frac{16}{9}, \\
\theta_{i}^{ \pm} & : \chi=\frac{1}{3} \vartheta\left[\begin{array}{c}
1 / 6 \\
0
\end{array}\right]+\omega^{i} \vartheta\left[\begin{array}{l}
1 / 6 \\
1 / 3
\end{array}\right]+\varpi^{i} \vartheta\left[\begin{array}{l}
1 / 6 \\
2 / 3
\end{array}\right], & h=\frac{1}{36}, \frac{25}{36}, \frac{49}{36},
\end{array}
$$

with $i=1,2,3$ and $\omega=e^{2 \pi i / 3}$. From the corresponding $S$-matrix we found the following fusion rules (all indices mod 3 ). First, the untwisted sectors give rise to the representation ring of $\hat{T} \cong S L(2,3)$

$$
\begin{aligned}
1_{i} \times 1_{j} & =1_{i+j}, \\
\phi_{i} \times \phi_{j} & =1_{i+j}+j, \\
j \times j & =2 j+\sum_{k} 1_{k} .
\end{aligned}
$$

Note that the last equation gives an explicit example of a $N_{i j k}>1$ with nondegenerate ground states, as can be explicitly checked using the above character formulas. The fusion rules of the twisted operators are

$$
\begin{aligned}
\sigma \times \sigma & =\tau \times \tau=\sum_{k} 1_{k}+j+\sum_{k} \phi_{k}+2 \sigma+2 \tau, \\
\sigma \times \tau & =2 j+\sum_{k} \phi_{k}+2 \sigma+2 \tau, \\
\omega_{i}^{+} \times \omega_{j}^{+} & =\theta_{i}^{+} \times \theta_{j}^{+}=\omega_{-i-j}^{-}+\sum_{k} \theta_{k}^{-}, \\
\omega_{i}^{+} \times \theta_{j}^{+} & =\theta_{i}^{+} \times \omega_{j}^{+}=\theta_{-i-j}^{-}+\sum_{k} \omega_{k}^{-}, \\
\omega_{i}^{+} \times \omega_{j}^{-} & =\theta_{i}^{+} \times \theta_{j}^{-}=j+1_{i-j}+\sigma+\tau, \\
\omega_{i}^{+} \times \theta_{j}^{-} & =\theta_{i}^{+} \times \omega_{j}^{-}=\sum_{k \neq i-j} \phi_{k}+\sigma+\tau .
\end{aligned}
$$


This can also be better understood if we consider the classes of $\operatorname{SL}(2,3)$. The 7 conjugacy classes are $1, \phi, \sigma, \omega^{ \pm}, \theta^{ \pm}$with elements of orders respectively $1,2,4,3$, 6 . The projections of the stabilizer subgroups are $\mathbf{Z}_{2}$ for $\sigma$, and $\mathbf{Z}_{3}$ for the elements $\omega^{ \pm}, \theta^{ \pm}$. The class algebra can again be recognized as overall selection rule in the fusion rules.

We can also consider this tetrahedron model as an abelian $\mathbf{Z}_{3}$ orbifold of the $\mathbf{D}_{2}$ model that we discussed before. The $\mathbf{Z}_{3}$ evidently permutes the operators $j_{a}, \sigma_{a}$ and $\tau_{a}$, which give rise to a single $j, \sigma$ and $\tau$ in the $T$ model. The fields 1 and $\phi$ are fixed points, and accordingly triple to form the representations $1_{i}, \phi_{i}$. Since we have two fixed points under an order three action, we expect $2 \times 6$ twist fields, which corresponds to the $\omega_{i}^{ \pm}$and $\theta_{i}^{ \pm}$. This gives indeed the total of 21 operators. If we mod out by another $\mathbf{Z}_{2}$ we obtain the 28 operators of the octahedral model. This analysis for the solvable groups can without much problem be extended to arbitrary level $k$.

\section{Appendix}

In this appendix we list some useful identities obtained in the theory of finite groups. To any finite group $G$ with elements $g_{i}$ we can associate a $\operatorname{set} \operatorname{Irr}(G)$ of irreducible representations $r_{\alpha}$ of dimension $d_{\alpha}$. The character of a representation is defined as

$$
\varrho(g)=\operatorname{Tr} r(g) .
$$

Useful identities are $\varrho\left(g^{-1}\right)=\varrho(g)^{*}$ and $\varrho(1)=\operatorname{dim} r$. The characters are invariant under similarity transformations $r(g) \rightarrow \operatorname{Sr}(g) S^{-1}$. This implies that they are only functions of the conjugacy classes $C_{A}$ and we can write $\varrho\left(C_{A}\right)$. The collection of conjugacy classes will be denoted by $\mathrm{Cl}(G)$. There are as many irreducible representations as there are (conjugacy) classes, although in general there is no natural mapping $r_{\alpha} \rightarrow C_{A}$. The fact that the $|\operatorname{Irr}(G)|=|\mathrm{Cl}(G)|$ can be proved by considering the group algebra.

The group algebra $A(G)$ (over $\mathbf{C}$ ) is the $|G|$-dimensional vector space with basis elements $g_{i} \in G$, i.e. it consists of elements $\alpha=\Sigma_{i} \alpha_{i} g_{i}, \Sigma_{i} \in \mathbf{C}$. Multiplication is defined as $\alpha \cdot \beta=\Sigma_{i, j} \alpha_{i} \beta_{j} g_{i} g_{j}$. The irreducible representations of $A(G)$ (as an algebra) are given by the elements of $\operatorname{Irr}(G)$. By the left or right action of $G, A(G)$ itself can be regarded as a representation, the so-called regular representation.

The center $Z(G)$ of $A(G)$ is a subalgebra spanned by the elements

$$
z_{A}=\sum_{g \in C_{A}} g \text {. }
$$

Now we can show using Schur's lemma that $r_{\alpha}(z)$ is a multiple of the identity, since it commutes with all elements $r_{\alpha}(g)$. Taking a trace we find the factor of proportionality

$$
r_{\alpha}\left(z_{A}\right)=\frac{\varrho_{\alpha}\left(C_{A}\right)}{\varrho_{\alpha}(1)} 1
$$

Upon further considerations one can show that this mapping is onto, so that $\mathrm{Cl}(G)$ can be considered as the dual space to $\operatorname{Irr}(G)$. 
The regular representation decomposes in $d_{\alpha}$ copies of the irreducible representation $r_{\alpha}$

$$
r_{\mathrm{r}}^{\mathrm{reg}}=\oplus_{\alpha} d_{\alpha} r_{\alpha}
$$

Taking traces of this identity we obtain the relation

$$
|G|=\sum_{\alpha} d_{\alpha}^{2}
$$

The characters of the irreducible representations satisfy some very useful orthogonality relations

$$
\begin{aligned}
\frac{1}{|G|} \sum_{g \in G} \varrho_{\alpha}(g h) \varrho_{\beta}\left(g^{-1}\right) & =\delta_{\alpha \beta} \frac{\varrho_{\alpha}(h)}{\varrho_{\alpha}(1)}, \\
\frac{1}{|G|} \sum_{\alpha} \varrho_{\alpha}(g) \varrho_{\alpha}\left(h^{-1}\right) & =\frac{1}{|C(g)|} \delta(g, h) .
\end{aligned}
$$

Here the $\delta$-function is defined by $\delta(g, h)=1$ if $g$ and $h$ are conjugate, and 0 otherwise. Both relations express the unitarity of the matrix

$$
S_{\alpha}^{A}=\left[\frac{\left|C_{A}\right|}{|G|}\right]^{\frac{1}{2}} \varrho_{\alpha}\left(C_{A}\right)
$$

The irreducible representations define an algebra

$$
r_{\alpha} \otimes r_{\beta}=\sum_{\gamma} N_{\alpha \beta}{ }^{\gamma} r_{\gamma}
$$

This is an associative, commutative algebra and accordingly has only onedimensional representations. They are given by the characters $\varrho_{\alpha}\left(C_{A}\right)$ as labelled by the classes. In particular there is one representation $\varrho_{\alpha}(1)=d_{\alpha} \in \mathbf{Z}_{>0}$. Accordingly we have

$$
N_{\alpha \beta \gamma}=\frac{1}{|G|} \sum_{g \in G} \varrho_{\alpha}(g) \varrho_{\beta}(g) \varrho_{\gamma}(g) .
$$

Acknowledgments. We acknowledge useful discussions with L. Dixon, P. Ginsparg, G. Moore, K. Schoutens, N. Seiberg and E. Witten. The work of R. D. is sponsored by the Stichting voor Fundamenteel Onderzoek (F.O.M.), that of C.V. by NSF contract PHY-87-14654, that of E.V. by NSF grant PHY-881752188, and that of H.V. by NSF grant PHY-80-19754.

\section{References}

1. Belavin, A.A., Polyakov, A.M., Zamolodchikov, A. B.: Nucl. Phys. B241, 333 (1984)

2. Friedan, D., Qiu, Z., Shenker, S.: Phys. Rev. Lett. 52, 1575 (1984)

3. Cardy, J.L.: Nucl. Phys. B 270, [FS16] 186 (1986)

4. Cappelli, A., Itzykson, C., Zuber, J.-B.: Nucl. Phys. B280 [FS18] 445 (1987); Commun. Math. Phys. 113, 1 (1987); Gepner, D., Nucl. Phys. B287, 111 (1987)

5. Verlinde, E.: Nucl. Phys. B300, 360 (1988)

6. Vafa, C.: Phys. Lett. 206B, 421 (1988)

7. Rehren, K.-H., Schroer, B.: Einstein causality and Artin braids. Berlin-preprint (April 1988) 
8. Mathur, S., Mukhi, S., Sen, A.: Differential equations for correlators and characters in arbitrary rational conformal field theories. Preprint TIFR/TH/88-32

9. Moore, G., Seiberg, N.: Phys. Lett 212 B, 451 (1988)

10. Dijkgraaf, R., Verlinde, E.: Modular invariance and the fusion algebra. Nucl. Phys. [Proc. Suppl.] 5B, 87 (1988)

11. Moore, G., Seiberg, N.: Naturality in conformal field theory. Preprint IASSNS-HEP/88/31

12. Moore, G., Seiberg, N.: Classical and quantum conformal field theory. Commun. Math. Phys. 123, 177-254 (1989)

13. Witten, E.: Quantum field theory and the Jones polynomial. Commun. Math. Phys. 121, 351399 (1989)

14. Goddard, P., Kent, A., Olive, D.: Commun. Math. Phys. 103, 105 (1986)

15. Dixon, L., Harvey, J.A., Vafa, C., Witten, E.: Nucl. Phys. B282, 620 (1985); Nucl. Phys. B274, 285 (1986)

16. Hamidi, S., Vafa, C.: Nucl. Phys. B279, 465 (1987)

17. Dixon, L., Friedan, D., Martinec, E., Shenker, S.: Nucl. Phys. B282, 13 (1987)

18. Vafa, C.: Nucl. Phys. B273, 592 (1986)

19. Frenkel, I. B., Lepowski, J., Meurman, A.: In Vertex operators in mathematics and physics. Publications of the Math. Sci. Res. Inst. No. 3. Berlin, Heidelberg, New York: Springer 1984

20. Dixon, L., Ginsparg, P., Harvey, J.: Beauty and the beast: superconformal symmetry in a monster module. Commun. Math. Phys. 119, 221 (1988)

21. Friedan, D., Shenker, S.: Nucl. Phys. B281, 509 (1987)

22. Vafa, C.: Phys. Lett. 199B, 195 (1987)

23. Narain, K.S., Sarmadi, M.H., Vafa, C.: Nucl. Phys. B288, 551 (1987)

24. See for example Gorenstein, D.: Finite groups. New York: Harper \& Row 1968

25. Lusztig, G.: Lcading cocfficients of character values of hecke algebras. In the Arcata Conference on Representations of Finite Groups. Fong, P. (ed.), Proceedings of Symposia in Pure Mathematics, Vol. 47 (1987); Characters of reductive groups over a finite field. Ann. of Math. Studies, Vol. 107. Princeton University Press 1984

26. Freed, D., Vafa, C.: Commun. Math. Phys. 110, 349 (1987)

27. Narain, K.S.: Phys. Lett. 169 B, 41 (1986); Narain, K.S., Sarmadi, M.H., Witten, E.: Nucl. Phys. B279, 369 (1987)

28. Dijkgraaf, R., Verlinde, E., Verlinde, H.: Commun. Math. Phys. 115, 649 (1988)

29. Ginsparg, P.: Nucl. Phys. B295 [FS21], 153 (1988)

30. Harris, G.: Nucl. Phys. B300 [FS22], 588 (1988)

31. Wakimoto, M., Yamada, H.: Lett. Math. Phys. 7, 513 (1983)

32. Dijkgraaf, R., Verlinde, E., Verlinde, H.: Conformal field theory at $c=1$. To be published in the proceedings of the 1987 Cargèse Summer School on Nonperturbative Quantum Field Theory

33. Dijkgraaf, R., Verlinde, E., Verlinde, H.: In Proceedings of the 1987 Copenhagen Conference. Singapure: World Scientific 1988

34. Bais, F.A., Bouwknegt, P., Surridge, M., Schoutens, K.: Nucl. Phys. B304, 348; 371 (1988)

Communicated by A. Jaffe

Received December 22, 1988; in revised form February 1, 1989 